\title{
Wind circulation in selected rotating magnetic early-B stars
}

\author{
M. A. Smith ${ }^{1}$ and D. Groote ${ }^{2}$ \\ 1 Space Telescope Science Institute/CSC, 3700 San Martin Dr. Baltimore, MD 21218 and Catholic University \\ of America, Washington, DC, USA \\ 2 Hamburger Sternwarte, Gojenbergsweg 112, 21029 Hamburg, Germany
}

Received 6 November 2000 / Accepted 21 March 2001

\begin{abstract}
The rotating magnetic B stars are a class of variables consisting of He-strong and some $\beta$ Cep stars which have oblique dipolar magnetic fields. Such stars develop co-rotating, torus-shaped clouds by channeling wind particles from their magnetic poles to circumstellar regions centered around the plane of their magnetic equators. The rotation of the cloud-star complex permits the study of absorptions from the cloud as it occults the star. In this paper we describe a quantitative analysis of archival $I U E$ data to map the properties of these clouds over four stars (HD 184927, $\sigma$ Ori E, $\beta$ Cep, and HR 6684). By computing spectral synthesis models for these stars, we find that only $\beta$ Cep has a solar-like metallicity. Our analysis also shows that the metal composition across the surfaces of all these stars is at least approximately homogeneous. Using the Hubeny code CIRCUS, we demonstrate that the periodic variations of broad-band ultraviolet continuum fluxes can be explained fully by the absorptions of the co-rotating cloud. We show next that among selected lines, those arising from low-excitation states are selectively affected by cloud absorption and turbulence. Our analysis also quantifies the cloud temperatures and column densities required to match the absorptions of a number of weak to moderate strength resonance lines. These temperatures increase with the ionization potential of the parent ions of these various lines, a result which is consistent with radiative equilibrium models in which temperature increases with proximity to the star's surface. Although these attributes appear stable from one epoch to another, dynamic processes are nonetheless at work. Both the strengths and widths of resonance lines at occultation phases indicate the presence of a turbulence in the cloud which increases inwards. The spectroscopic hallmark of this stellar class is the presence of strong CIV and $\mathrm{N}$ V resonance line absorptions at occultation phases and of redshifted emissions of these lines at magnetic poleon phases. The emissions have characteristics which seem most compatible with their generation by high-energy shocks at the wind-cloud interface, as predicted recently by Babel (1998).
\end{abstract}

Key words. stars: individual: $\beta$ Cephei - stars: individual: HR 6684 - stars: individual: $\sigma$ Ori E - stars: individual: HD 184927 - stars: chemically peculiar - stars: magnetic fields

\section{Introduction}

The He-strong Bp stars are a subclass of early-type B stars with an array of remarkable properties, chief among which are their oblique dipolar magnetic fields, magnetically controlled winds, and chemical surface anomalies (e.g. Bohlender et al. 1987; Bohlender 1994; Bolton 1994; Shore 1998). These conditions provide a rich testbed for the study of magnetic and hydrodynamical processes on these stars and conceivably on stars of related active Oand B-subclasses in which wind and photospheric line variability has so far gone undiagnosed. Several seminal papers on the He-strong star $\sigma$ Ori E have demonstrated that several peculiarities in the ultraviolet spectrum of this star can be explained by circumstellar clouds forced into co-rotation over the magnetic equator. For example,

Send offprint requests to: M. A. Smith,

e-mail: msmith@stsci.edu
Groote \& Hunger (1976) showed that high-level Balmer lines appear at certain rotational phases, demonstrating the presence of an occulting, comparatively low-density gas. This circumstellar medium can also be observed as periodically modulated $\mathrm{H} \alpha$ emissions (e.g., Short \& Bolton 1994).

Using spectra from the International Ultraviolet Explorer (IUE) satellite, Shore and collaborators (Shore \& Brown 1990 (SB90); Shore et al. 1990) demonstrated that a common property of winds in both He-strong and He-weak Bp stars is that they are channeled by a dipolar magnetic field to a torus-shaped magnetosphere (cloud) located over the magnetic equator. This torus may well be located in a different volume than the plasmasphere suggested by radio studies (Havnes \& Goertz 1984; Drake et al. 1987; Drake 1998), but it is likely to be related. Following the initial ideas of Shore (1987); Babel \& Montmerle (1997a) and Babel (1998) have discussed 
a model in which shocks are excited at the wind-torus interface, creating high energy emission. The magnetic and rotational axes of these stars are separated by seemingly arbitrary angles. In most cases this angle is large enough to provide an opportunity to observe the timevariability of emission and absorption components of the strong lines and thus to study the azimuthal structure of the clouds as the wind-cloud complex co-rotates. While these variations are often large and complex, all indications are that they are rather stable over decades (e.g., SB90, Bolton 1994; Henrichs et al. 2001).

Henrichs et al. (1998) recently proposed the existence of a distinct new class of magnetic rotators (hereinafter, rotating magnetic $B$ stars). The three stars so far assigned to this group, $\beta$ Cep (HD 205021), HR 6684 (HD 163472), and HD 184927, are characterized by redshifted emissions of the C IV resonance lines. To this small class, we would add the extensively studied, He-strong star $\sigma$ OriE. The Si IV and CIV resonance lines of this star also show redshifted emissions at phases of magnetic extrema (Bohlender et al. 1986). We have examined the sequences of $\mathrm{CIV}$ and $\mathrm{NV}$ resonance profiles in three He-strong stars in the SB90 sample with known rotational periods (HD 37017, HD 37776, HD 64740) and find that each exhibits phase-correlated redshifted emissions. An examination of NEWSIPS-processed spectra of HD 36385 and HD 133518 shows that the CIV resonance lines in these stars' spectra also exhibit variable, redshifted emissions. The periodic emissions of the $\mathrm{NV}$ and $\mathrm{CIV}$ lines of the hot O7 V star $\theta^{1}$ Ori C are well known. Walborn \& Nichols (1994) and Gagné et al. (1997) have suggested that this star is a hot analog to the Bp stars. Because of the similar variabilities of the red halves of their C IV, N V profiles, it is likely that these stars, and indeed perhaps all He-strong variables, should be included in this new class.

In this paper we provide the first detailed analysis of spectral lines to determine conditions in co-rotating clouds attached to the rotating magnetic B stars. We undertake this study by using ultraviolet lines obtained entirely from archival echellograms obtained with the IUE satellite. We have three specific goals: (1) to estimate physical parameters of clouds (temperature, density, extent, metallicity, and turbulence), (2) to study the relevance of Bp-like surface chemical composition (metal-depletion) on cloud properties, and (3) to examine the processes responsible for resonance line emissions and anomalous absorptions at certain phases.

For these purposes we have chosen the first four stars discussed above: $\sigma$ Ori E (the most well studied He-strong star), HD 184927 (a sharp-lined, magnetic He-strong star; Wade et al. 1997), $\beta$ Cep (a composition-normal star with magnetic wind properties similar to the He-strong stars; it is also the prototype of the $\beta$ Cephei pulsational variables), and HR 6684 (a $\beta$ Cephei variable with a moderate rotational velocity; Jerzykiewicz 1972; Kubiak \& Seggewiss 1984). These last two stars are pulsators and are not He-strong stars. The selection of these four stars was guided in part by our desire to include both intrinsically slow ( $\beta$ Cep) and moderately rapid rotators ( $\sigma$ Ori E and HR 6684), as well as stars observed at both low and high aspect angles. $\beta$ Cep and $\sigma$ Ori E are probably intrinsically moderate and rapid rotators, respectively, viewed nearly equator-on. HD 184927 is an intrinsically slow rotator viewed at low inclination. Less is known about HR 6684, but its low $v \sin i$ and possible rotation period near 3.75 days (Henrichs et al. 1998), suggest that it is a moderate rotator observed at low inclination, an interpretation supported by the single-wave variations of its magnetic and optical He I line variations (Wade et al. 1997). In order to describe the geometry of the magnetospheres, it is important that the sample of stars studied has a variety of rotational velocities and be viewed from a range of inclination angles.

\section{Reduction and analysis of archival IUE data}

\subsection{Data reduction}

The IUE data for this program were the NEWSIPSextracted, large-aperture high dispersion spectral files from the Short Wavelength Prime (SWP) camera. These were downloaded from the MAST ${ }^{1}$ data archive. The data used were all the IUE SWP/large-aperture images for $\sigma$ Ori E (29), HD 184927 (30), $\beta$ Cephei (31), and HR 6684 (41), except that for $\sigma$ Ori E the images SWP 04840 and SWP 27259 were excluded. The latter spectra were observed at isolated times and thus do not lend themselves to analysis of short-term variations. The high-dispersion SWP-camera data for both NEWSIPS and IUESIPS are known to depend on errors in echelle ripple correction and background subtraction. Ripple-correction errors were avoided by working with net flux data for individual echelle orders. To account for background extraction errors (Smith 1999), we derived corrections first in the $\beta$ Cep and HD 184927 spectra by forcing the depths of the Si III, Si IV, C IV, and occasionally other resonance lines to zero. This procedure was generally insensitive to rotation because most of the resonance lines we studied reside well up on the flat or even damping parts of their curves of growth, and their line cores are well broadened by saturation. The zero-point corrections we derived were typically $5-10 \%$ of the continuum level. For lines in shortwavelength orders, we interpolated corrections from the Ly $\alpha$ and other nearby orders.

\subsection{Computation of simulated spectra and spectral absorptions}

We used a suite of codes written by I. Hubeny and collaborators to compute model line profiles to compare with the IUE data. The first code is SYNSPEC, a spectral line synthesis code developed for input non-LTE or lineblanketed model atmospheres by Hubeny et al. (1994).

\footnotetext{
1 Multi-Mission Archive at Space Telescope Science Institute, in contract to NASA.
} 
We used standard LTE atmospheres by Kurucz (1993) in our work. SYNSPEC is embedded within an IDL wrapper (Hubeny 1996) such that we could easily calculate spectra with a variety of trial atmospheric abundances. SYNSPEC was then run to generate synthetic spectra similar to the observed ones by computing a disk-integrated, rotationally broadened spectrum and to convolve it to the spectral resolution (13000) of high dispersion IUE spectra.

Our principal objective in this paper is to assess the effect of torus-shaped magnetospheric clouds on a star's spectral lines (Shore \& Brown 1990) as they alternatively move onto and off the projected stellar surface. To simulate the signatures of a cloud on the composite spectrum, we used the Hubeny CIRCUS program (Hubeny 1996; Hubeny \& Heap 1996). This code was written to compute line absorptions and/or emissions of a gas cloud situated either in front or off the limb of a reference star. CIRCUS requires the user to specify physical cloud parameters such as temperature, density, geometry, composition, microturbulence, column depth, and areal coverage factor. Although the code can accommodate as many as three separate clouds we considered only a single homogeneous cloud in nearly all our simulations. In its solution of the radiative transfer equation within a cloud, CIRCUS computes line emissions and absorptions separately. This feature can be used to compute the cloud spectrum either in LTE (adding differential emission and absorption terms) or in the two-level non-LTE approximation, in which only the absorption terms are calculated.

The code proceeds by first consulting a Kurucz (1990) line library of atomic absorption parameters and computing an opacity spectrum for a user-specified temperature and electron density. The spectra were computed at a spacing of 0.01 Angströms, which oversamples the contributing lines. The optical depth in each line can then be determined using an input column density. The surface of the star is divided into a $100 \times 100$ grid and the local intensity spectrum is evaluated at each grid point, thereby taking into account the effects of foreshortening and limb darkening. Typically, we computed CIRCUS models in pairs. In the first case we assumed that a square cloud occults the stellar disk. In the second, we allowed the position of the cloud to shift off the projected stellar disk so that it contributed only line emission to the spectrum. The dimensions of real clouds near a magnetic B star are actually likely to be much larger than one stellar radius (Hunger et al. 1990; Bolton 1994). Thus, we expect that our treatment underestimates the total effects of emission at non-occultation phases. However, since we modeled only differences in line strengths between occultation and nonoccultation phases, this fact is not relevant to our analysis.

The density and areal coverage factors of the cloud are necessary but not critical parameters in our analysis. For our initial models we started with a trial volume density of $10^{12} \mathrm{~cm}^{-3}$, based on the appearance of high Balmer series members during the occultation phases of $\sigma$ Ori E (Groote \& Hunger 1976; Short \& Bolton 1994). From the resulting column densities (Sect. 4.3) and an estimate of $6 R_{*}$ for the extent of the cloud in the line of sight from these authors, we derived a mean density of $\sim 3-5 \times 10^{10} \mathrm{~cm}^{-3}$. As a compromise we chose a density of $1 \times 10^{11} \mathrm{~cm}^{-3}$, which we used in our models. The results of this study are not very sensitive to the electron density for the ranges considered.

CIRCUS includes a provision for various doppler effects. The most important of these is stellar rotation, which CIRCUS treats by shifting the local intensity spectrum by a wavelength equivalent to the projected doppler shift at each point on the disk. The program computes the net doppler velocity between each projected element of the cloud and the background star along the observer's line of sight. In our model there are no differential velocities between regions of the cloud along the line of sight and the projected area on the star because the cloud is corotating. Additionally, because microturbulence can be an additional factor in determining the signatures of a cloud on a spectrum, we varied this as a free parameter. In general, values less than $20 \mathrm{~km} \mathrm{~s}^{-1}$ resulted in similar cloud absorption/emission spectra, so we used this value as a default.

\section{Basic picture}

To date only a few models of the dynamics of rotating magnetic B stars have been discussed for He-variable stars. Of these most work has focused on $\sigma$ Ori E. Borrowing from work of Groote \& Hunger (1976, 1982, 1997; Hunger \& Groote 1993; Hunger et al. 1990); Shore \& Brown (1990), Babel \& Montmerle (1996, 1997a), Babel (1998), this prototype and probably all He-variable stars exhibit variations because they have dipolar fields which are inclined to some extent with respect to their magnetic axes. There is now a broad consensus that closed magnetic loops from the two poles divert a weak wind emerging from these surface regions. The particles emanating from opposite poles collide at high velocity and form a torus-shaped magnetosphere. The particles eventually leak out through the outer edge of the torus (defined by the Alfvén radius) and also settle back to the star - to what relative extents is still unclear.

The radiative wind theory of Springmann \& Pauldrach (1992) suggests that there is a tendency in this region of $T_{\text {eff }}, \log g$ space for $\mathrm{H}$ and $\mathrm{He}$ atoms to separate during their flow. This chemical fractionation is one of several complex feedback processes which determines the evolution of the wind. In our picture a relative preponderance of metal ions are directly accelerated by the magnetic polar wind along open field lines and leaves the star permanently. At the same time many helium atoms are decoupled early in the flow (as is hydrogen in cooler stars) and return back to the surface along roughly the lines of force that guided their upward flow. At the surface Bp-like chemical anomalies (He-richness, metal-deficiency) establish local vertical abundance gradients over time unless they are destroyed, e.g. through mixing. Wind particles guided along closed lines accumulate in the torus for a short time before they are lost at their edges. The length 
of time these particles spend in the torus is far shorter than the evolutionary lifetime of the star (e.g., Groote \& Hunger 1982; Havnes \& Goertz 1984; Linsky et al. 1992), so the torus's composition will mirror the abundances found at the star's magnetic poles.

The above description is meant only to lay out a broad picture that is pertinent to this paper. Havnes \& Goertz (1984) and Linsky et al. (1992) have discussed dynamic models of a magnetic plasmasphere in which nonthermal electrons emit gyro-synchrotron radiation in a current sheet some 10-20 $R_{*}$ from the star (Phillips \& Lestrade 1988). These models were conceived in part because of the abnormally high radio fluxes of these stars (Linsky et al. 1992). In contrast, X-ray observations do not suggest an abnormal level of high energy emission (Drake et al. 1994). This same comment applies to the strength of the wind components of the ultraviolet resonance lines. Moreover, at a given rotational phase the variability patterns of the ultraviolet and optical lines appear very similar from one epoch to another at any given rotational phase. For these reasons the volumes emitting fluxes in these different wavelength regimes are probably not cospatial. Thus, the physics of this emission is likely to be quite different. In this paper our philosophy will be to infer kinetic and thermodynamic properties of the magnetospheric torus-clouds within several stellar radii using the ultraviolet and optical data alone. Additionally, we will be reluctant to invoke magnetic (or rotational) energy sources or sinks unless needed.

Our examination of these questions proceeds by considering first the obscurations of the photospheric spectrum by the torus-cloud. Since the clouds are locked into co-rotation with the surface, the photospheric and cloud contributions of a line profile cannot be separated according to differences in their shapes. Instead we must rely on the modulation of the cloud's absorptions when it occults the star once or twice each rotational period. Before proceeding to this analysis, we first discuss the relevant parameters required to compute the underlying photospheric line spectrum.

Ultraviolet studies have shown that the He-strong stars are on or just evolving off the main sequence (e.g., Shore 1990) and hence have $\log g$ values in the range 3.5-4.0. We chose $T_{\text {eff }}$ values for our program stars from relevant papers in the literature and list them in Table 1. Rotational periods and magnetic ephemerides are well known for these stars (except for HR 6684) and are listed as well.

\section{Spectral analysis of line absorptions}

\subsection{The photosphere: Metallicity and $T_{\text {eff }}$}

Hunger et al. (1990) and Reiners et al. (2000) concluded that the metal abundances on the surfaces of $\sigma$ Ori E, are low at the magnetic poles and roughly solar-like over the magnetic equator. However, their analyses were performed on optical spectra with the implicit assumption that the effects of CII, SiII line absorptions from the
Table 1. Adopted atmospheric parameters. Notes: (1) $T_{\text {eff }}$ is given in $\mathrm{K}, v \sin i$ in $\mathrm{kms}^{-1}$, and the zeropoint of rotational phase (North magnetic pole crossing) as HJD + 2400000. (2) $\Phi=0.0$ for HR 6684 might be quite inaccurate. (3) Sources are given as indices: 1: Groote \& Hunger (1997), 2: Wade et al. (1997), 3: Gies \& Lambert (1992), 4: Bolton et al. (1986), 5: Campos \& Smith (1980), 6: Hesser et al. (1977), 7: Henrichs et al. (2001), 8: Henrichs et al. (1998).

\begin{tabular}{l|c|c|c|c}
\hline \hline & $\sigma$ Ori E & HD 184927 & $\beta$ Cep & HR 6684 \\
\hline$T_{\text {eff }}$ & $23000^{1}$ & $23000^{2}$ & $27000^{3}$ & 26000 \\
$\log g$ & 4.0 & 4.0 & 4.0 & 4.0 \\
$v \sin i$ & $162^{4}$ & $15^{2}$ & $30^{5}$ & 100 \\
$P_{\text {rot }}$ & $1.1908106^{6}$ & $9.52961^{2}$ & $12.00092^{7}$ & $\approx 3.75^{8}$ \\
$\Phi=0.0$ & 44241.537 & 49987.388 & 51238.15 & 49953.03 \\
\hline
\end{tabular}

magnetospheric clouds are negligible. The Reiners et al. metallic-line strength curves show the same offset in phase with respect to the magnetic pole crossing phase that our Fig. 4a does, suggesting that enhanced optical and ultraviolet absorptions are formed in the same locality. We tested this possibility by constructing additional CIRCUS models for the optical C II and Si II wavelengths and found that these were able to reproduce fractional equivalent width changes of $10-20 \%$. Thus we are led to a contrary picture, namely that the variation of UV and optical lines and the continuum is due to absorptions within a co-rotating cloud.

Our line synthesis analysis was conducted by first selecting rotational phases for which line strengths in our IUE data were minimal and then using SYNSPEC to match spectra in the 1853-1876 and 1906-1931 Angström regions. We chose $\beta$ Cep as a control star and discovered that the lines can be nearly similarly matched by model atmospheres having either a normal metal abundance and a high effective temperature $(27000 \mathrm{~K})$ or a metallicity of one-tenth the solar value and a lower temperature $(24000 \mathrm{~K})$. We were able to break this ambiguity by modeling the wings of the Si III $\lambda 1206$ and Si IV $\lambda 1394, \lambda 1403$ lines only with the higher temperature model. This discrimination is possible because the ionization of $\mathrm{Si}^{2+}$ and hence the strength of $\lambda 1206$ is more sensitive to $T_{\text {eff }}$ than to metallicity. The high-temperature, normal-abundance model leads to less extended wings for $\lambda 1206$. Our adopted value $T_{\text {eff }}=27000 \mathrm{~K}$ (and normal composition) agrees well with the value $26600 \mathrm{~K}$ found by Gies \& Lambert (1992). Figure 1a shows overplots of an IUE spectrum in the region of the $\mathrm{Al}$ III doublet with both the adopted and the $24000 \mathrm{~K}$ synthesized models.

Following the same procedure, we estimated the metal abundances for a minimum-absorption observation (SWP 55488) of HR 6684 in the AlIII and Fe III wavelength regions. As with $\beta$ Cep, we found a trade-off between composition and stellar effective temperature, but this time both a low abundance and high $T_{\text {eff }}$ was needed to simulate the comparatively weak line strengths. As before, the weak wings of the SiIII and SiIV 

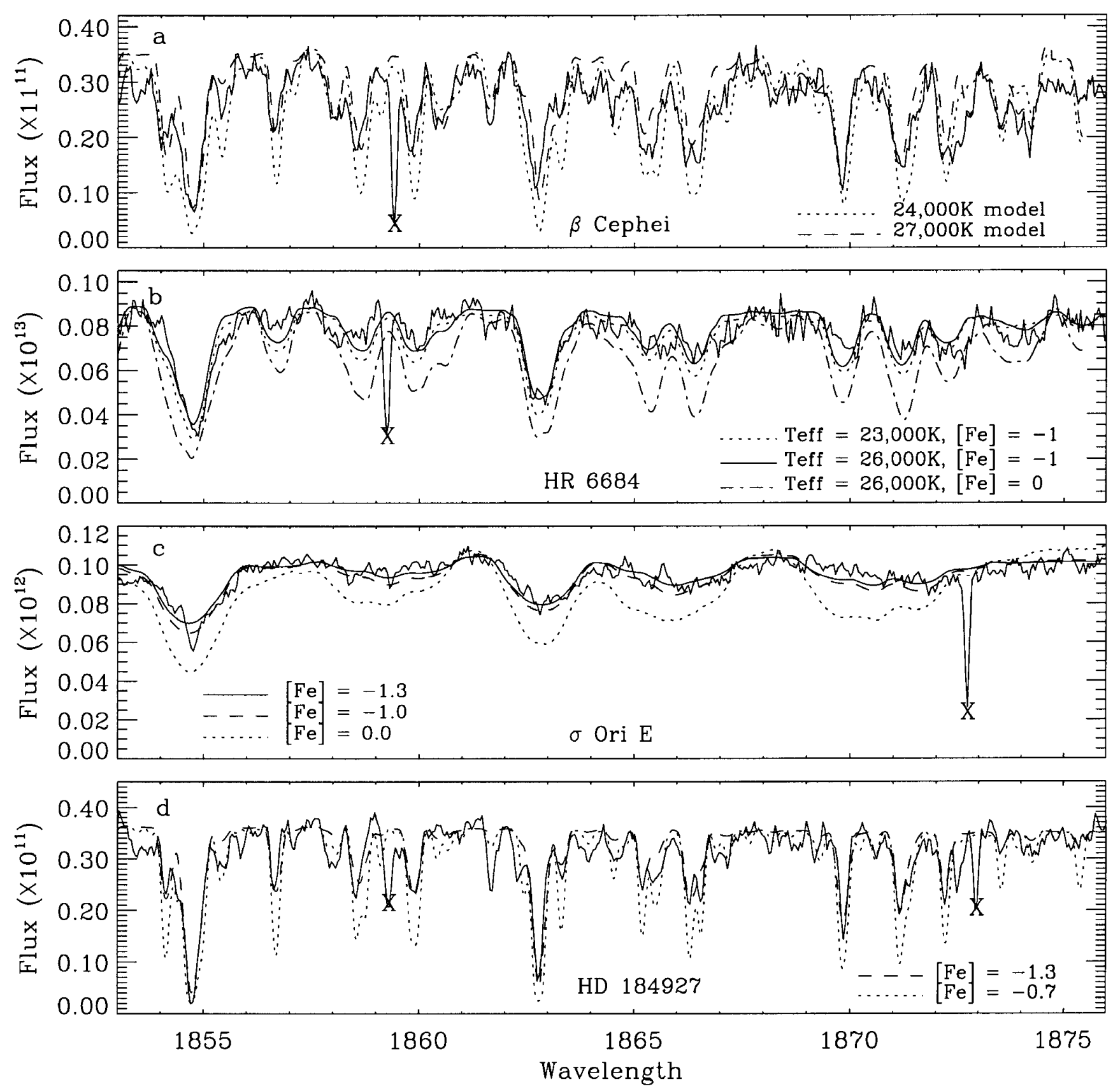

Fig. 1. a-d) Comparison of $I U E$ observations with $S Y N S P E C$-synthesized spectra of the wavelength region surrounding the AlIII doublet. The observations were made near the phase when the North magnetic pole crossed the stellar meridian: a) for $\beta \mathrm{Cep}$, spectrum SWP 52432 is compared with SYNSPEC models having $T_{\text {eff }}=27000 \mathrm{~K}$, $[\mathrm{Fe}]=0$, and $T_{\text {eff }}=24000 \mathrm{~K}$, $[\mathrm{Fe}]=-1, \mathrm{~b}$ ) for HR 6684 (at line minimum; no field detection yet) spectrum SWP 55488 is plotted with models having $T_{\text {eff }}=26000 \mathrm{~K},[\mathrm{Fe}]=0,-1$, and $\left.T_{\text {eff }}=23000 \mathrm{~K},[\mathrm{Fe}]=-1, \mathbf{c}\right)$ for $\sigma$ Ori E, five spectra (SWP 07555-6, 15785-7) are compared with models having $T_{\text {eff }}=23000 \mathrm{~K},[\mathrm{Fe}]=0,=-1 \&-1.3, \mathbf{d}$ ) for HD 184927 four IUE spectra (SWP 14504, 14511, 55612, $55770)$ are compared with $T_{\text {eff }}=26000 \mathrm{~K},[\mathrm{Fe}]=-0.7 \&-1.3$; the latter model fits so well it is largely hidden by the observed spectrum.

resonance lines at the non-occultation phases confirm this model. The fitting ambiguity was again settled in favor of high temperature $(26000 \mathrm{~K})$ but a low metal abundance. Figure $1 \mathrm{~b}$ exhibits the comparison for both low $([\mathrm{Fe}]=$ $-1)$ and normal metallicty and this effective temperature as well as a low-metals, high temperature model. The metal lines for the normal-abundance, low-temperature case are of course even stronger. For both effective temperatures the normal abundance model can be ruled out. For HR 6684 we estimate a metallicity $[\mathrm{Fe}]=-1.0 \pm 0.3 \mathrm{dex}$ (internal errors).

We digress to point out that because helium-rich atmospheres have comparatively low continuous opacities, a synthesized spectrum matched to observed metal line 
strengths will tend to err on the high side of the true photospheric metallicity. However, such errors are likely to be small for the mild helium overabundances of $2-3 \times$ as occur on most He-strong stars. A similar assessment can be made of the effects of magnetic fields to the measured line splitting, either due to Zeeman splitting or to the modification of the atmospheric structure. Such effects could produce in principle a line strengthening when the magnetic poles are visible and thus would produce small-amplitude line strengthenings at this phase. Note that such effects go in the wrong direction to explain the general line-strengthening during the occultation phase.

Our fits to lines of the He-strong stars $\sigma$ Ori E and HD 184927 were conducted by using the effective temperatures, $23000 \mathrm{~K}$, from previous optical studies. For these stars the abundances refer explicitly to the magnetic polar caps. In Fig. 1c we depict the synthesized spectrum in the AlIII line region with a low-absorption observation of $\sigma$ OriE at $\phi \approx 0.8$, that is, between the crossings of the North magnetic pole and the associated He-spot. For broad-lined spectra such as these, the accuracy of an abundance determination deteriorates. Nonetheless, our comparisons show a preference for the $[\mathrm{Fe}]=-1 \pm 0.4$, or less. Thus, our results are in mild disagreement with the optical result with the Hunger et al. (1990) finding of a metal deficiency of only a factor of 2.5 but are comparable to the Reiners et al. (2000) results. The program star, HD 184927, provides an optimal opportunity for line synthesis comparisons because its photospheric lines are virtually unbroadened. Figure 1d exhibits a match with an average of four IUE spectra in the AlIII line region with a metal abundance $[\mathrm{Fe}] \approx-1.3 \pm 0.3$.

We now consider line formations in the clouds, starting first with the weak metallic line absorptions formed at great distances from the star and will then work our way inwards.

\subsection{Comparison of time-variable spectra}

Another approach to disentangling the spectra of the cloud and photosphere is by means of the Temporal Variance Spectrum (TVS). Its use opens the question of whether heterogeneous metal abundances across the star's surface contribute appreciably to the observed variation of metallic line strengths. This spectrum permits a comparison of each line's activity with its mean strength. If photospheric abundances change from point to point on the star, the absorptions of all lines will be affected to some degree. On the other hand, if excess line absorptions occur in a cool circumstellar cloud at some distance from the star, low-excitation (and usually strong) lines are those we expect primarily to show fluctuations in the TVS.

Figures 2d,e test this expectation for HD 184927. Because of its slow rotation this star offers the best case for detecting the variability of individual lines. In this figure we display two spectral orders containing prominent $\mathrm{Al}$ III and Fe III lines. Because the excitation potentials of the lower levels of several other Fe III (and TiII) lines are high, we give these values as well. The lower (TVS) plot shows that only those lines with excitations of a few $\mathrm{eV}$ or less exhibit significant TVS fluctuations. Figure 2c shows the same pattern for the Al III line region of $\sigma$ Ori E. Clearly, all or nearly all the variations of the metal lines arise in a cool medium. Inspection of the line depths confirms that they arise one-quarter of a cycle earlier or later than the transits of the star's meridian.

To place limits on the variation of metals on the surface of HD 184927, we chose three IUE spectra obtained at faint-star (magnetic null) phases and compared line synthesis models containing weak, high excitation lines. Our best fitting models for the spectra in the two orders containing the AlIII and Fe III lines suggested a metallicity of $[\mathrm{Fe}]=-1.0 \pm 0.3$ (internal error), which is only marginally different from the metallicity of -1.3 estimated from Fig. 1d. The reader can discern the line activity from the lower line in Fig. 2d, which is the TVS spectrum of all observations. Note that the Al III lines themselves still have a residual absorption, which we interpret as due to the cloud. Otherwise, the differences for most other (high$\chi)$ lines are generally nil. Plots for the spectrum in the Fe III $\lambda 1914$ region show similarly small differences. We conclude that essentially all the UV line variations are due to absorptions from occulting clouds. Supporting evidence for this conclusion comes in addition from near-UV and visible-band data for $\sigma$ Ori $\mathrm{E}$ discussed in the next section.

As Fig. 2 shows, the TVS profiles have double lobes. To investigate the cause of these structures, we averaged two subgroups of spectra observed at phases of maximum and minimum strengths (hereafter referred to as clear-star and occultation phases). The low-excitation lines in the clearstar spectra have triangular shapes and narrow cores in these plots whereas the occultation-phase spectra exhibit broad, rounded cores. We confirmed that these differences in shapes arise during particular phases by inspecting individual spectra and seeing the patterns in them. Because the cloud-tori are the presumed products of a rapid deceleration of wind particles, it is natural to attribute the excess line broadening as a part of the deceleration process - a turbulence of $25-50 \mathrm{~km} \mathrm{~s}^{-1} .^{2}$

\subsection{The cloud spectrum and derived cloud parameters}

CIRCUS was used to compute the absorption of the stellar flux by the cloud (the so-called iron curtain) by subtracting the photospheric spectrum, computed by $S Y N S P E C$ from the photospheric plus cloud spectrum computed by CIRCUS. This was done for a variety of trial

\footnotetext{
${ }^{2}$ It is conceivable that this broadening could instead have a magnetohydrodynamic origin, e.g. from the generation of Alfvén waves by motions of field lines. However, for a dipolar field in a weak-field star like $\beta$ Cep the velocities of such waves would probably be smaller than these values. Moreover, Alfvén waves would produce motions transverse to the line of sight when the torus occults the star.
} 

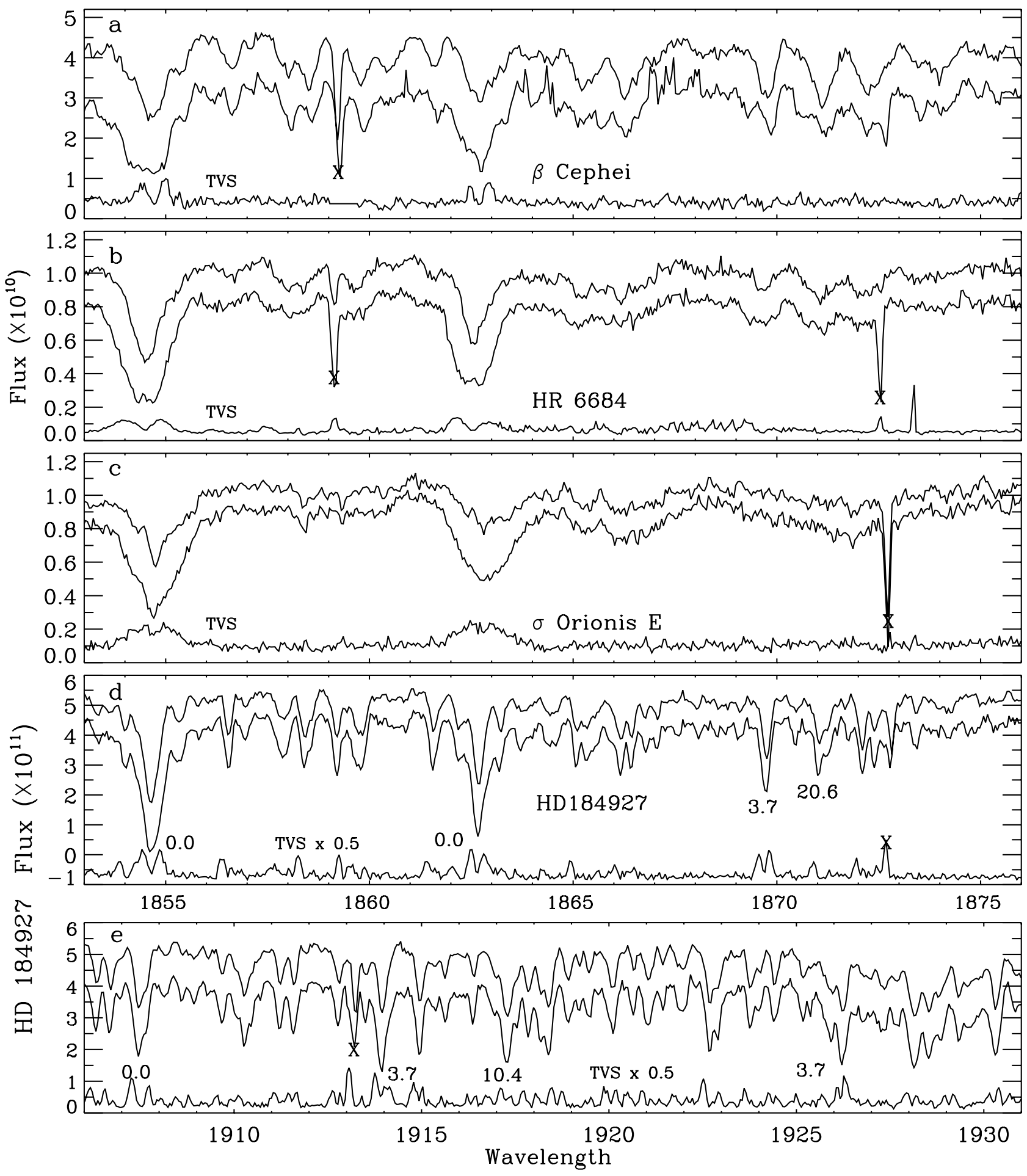

Fig. 2. a-e) Comparison of spectra in AlIII doublet region for program stars; clear star and occultation phases are shown in the top and lower spectrum (spectra offset for clarity). The TVS spectrum is shown at the bottom for the ensemble of spectra: a) for $\beta$ Cep, upper spectrum is from SWP 06234, 42939, \& 52620, and the lower one is from SWP 46244, 46255, 52121, 52460, 52573 , \& 52594, resp., b) for HR6684, upper spectrum is from SWP 14514, 55668, 55672, \& 55732, c) for $\sigma$ OriE upper spectrum is from SWP 07553, 07555, and the lower one from SWP 07534, 07536-7, 07560, 07583, \& 07609, d) for HD 184927 the upper spectrum (top) is from SWP 14468, 14475, 14487, 55669, 55685, 55701, and the lower one is from SWP 14504, 14511, $14681,55629,55770, \& 55781$ (excitations are given for a few lines), e) for HD 184927 as in d) but for the wavelength range surrounding low-excitation Fe III lines.

temperatures, column densities, and occasionally other parameters such as volume density, microturbulence and areal coverage factor. In general, the lower the cloud temperature, the higher the monochromatic absorption 
because of the increased influence of hydrogen opacity. Figure 3 a shows a comparison of the ratio of $I U E$ spectra obtained at the maximum and minimum flux phases for $\sigma$ Ori E. The principal features of interest in this figure are the increased absorption below 1300 Angströms and also near $\lambda 1900$. Our tests showed that these wavelengthdependent features can be reproduced by having cool temperatures, viz. $T_{\text {cloud }}=11500-13000 \mathrm{~K}$. In Fig. 3b we show a fit with similar parameters to observations of $\beta$ Cep taken from clear-star and occultation phases of the rotation cycle (but at similar pulsation phases). For both stars these cloud temperatures are consistent with radiative equilibrium temperatures expected for material situated several stellar radii from the surface (e.g. Drew 1989; Millar \& Marlborough 1999). We also experimented with two-zone cloud models. Because inner-cloud temperatures are likely to be higher in these models, and the opacities lower, the total cloud opacities at a given wavelength tend to be much as the same as for one-zone, lowtemperature models. As a result, we found that for the weak Fe-curtain one cannot distinguish very well between judiciously chosen multiple temperature and single lowtemperature models.

As an additional test of the models for Fig. 3a, we reran the models for the $\lambda \lambda 3700-4000$ range used by Groote \& Hunger (1976) to show that the torus occultation is accompanied by increased core absorption of the high-level Balmer-lines. Our models (not shown) exhibit similar line strengthenings as those observed (20-30\%). This result further validates the interpretation that the line strength variations arise from absorptions in the cloud.

To place our spectroscopic results in a context in which they can be compared with previous photometric studies, we summed the fluxes in four IUE echelle orders in the uninterrupted wavelength range $\lambda \lambda 1800$ 1905 and plotted them as a function of time. The resulting continuum ( $U V C$ ) light curves for $\sigma$ OriE and $\beta$ Cep in Figs. 4a,c (top curves) exhibit a pair of light dips corresponding to cloud occultations. The stronger of the two dips is $14 \%$ for $\sigma$ Ori E and $11.5 \%$ for $\beta \mathrm{Cep}^{3}$. We estimate the corresponding flux dips for HR 6684 and HD 184927 to be $2 \pm 0.7 \%$ and $3-4 \pm 1 \%$, respectively, i.e., just barely detectable. Some interesting conclusions can be drawn about the chemical composition of the $\sigma$ OriE cloud by comparing the amplitude of the primary dip in this star's UVC light curve, $14 \%$, with the $\approx 11 \%$ variation in the Strömgren $u$-band (Hesser et al. 1977). To see what cloud conditions could make the $I U E$ - and ground-based absorptions of $\sigma$ Ori E consistent with one another, we computed CIRCUS models in the $\lambda \lambda 1800$ 1905 and $\lambda \lambda 3450-3650$ (roughly, Strömgren-u) regions. Several cloud parameters were varied, including temperature, metallicity, turbulence, and column density. All our

\footnotetext{
3 The narrowness in phase of the dips for $\sigma$ Ori E may eventually turn out to be important in constraining the thickness of the torus in the direction of the magnetic axis, at least at several stellar radii from the star.
}

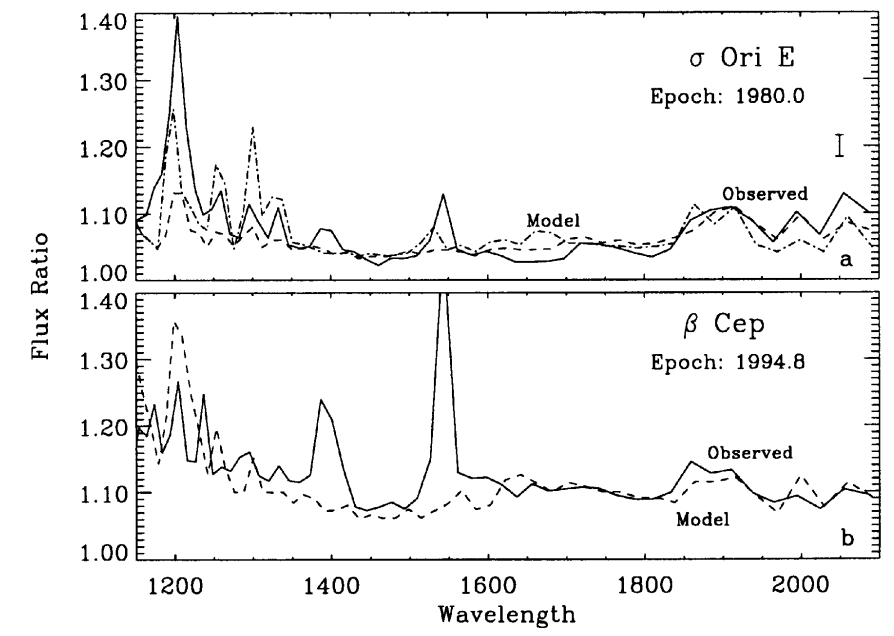

Fig. 3. a and b) Observed and computed ratios of (quantity +1 ) cloud absorption spectra for $\sigma$ OriE and $\beta$ Cep. a) For $\sigma$ Ori E the observed curve (thick solid line) is the ratio of IUE spectra SWP 07555-6, 07558, $07560(\phi=0.40-0.59)$ to SWP 07583 ( $\phi=0.02$, observed several hours earlier), binned one point per echelle order. Dot-dashed and dashed line curves denote $C I R C U S$ models with $T_{\text {cloud }}=11500 \mathrm{~K}$ and $13000 \mathrm{~K}$, with column densities of $6 \times 10^{22}$ and $1 \times 10^{23}$, and with coverage factors of $100 \%$ and $50 \%$, resp. The cloud spectra represent the fractional absorption plus one (in continuum units). b) For $\beta$ Cep the observations are taken from the ratio of SWP 52415,52594 to SWP 52514, 52653. The model has parameters $T_{\text {cloud }}=11500 \mathrm{~K}$, a column density of $1 \times 10^{23}$ particles $\mathrm{cm}^{-2}$, and a $70 \%$ coverage factor.

trials predicted larger absorptions for $\lambda \lambda 1800-1905$ region than for the Strömgren- $u$ region. The most important result of these tests was that the absorption ratio of these wavelengths was found to be highly sensitive to metallicity. For example, the ratio decreases markedly from 2.0 at normal metallicity to 1.4 for $[\mathrm{Fe}]=-1$. This sensitivity can be explained as follows: the fluxes of a B star in the $u$-bandpass are almost independent of metallicity because they are determined almost entirely by Balmer bound-free opacity. In contrast, in low-metal models the contributions of hydrogenic and metal line opacities are roughly equal in the $\lambda \lambda 1800-1900$ region. Since the great majority of the metal lines in this wavelength region are unsaturated, their absorptions vary almost linearly with metallicity. Taking our computed ratio of 1.4 for the lowmetals case, these results suggest that observed $u$-band variations should be about $1.4 \times 0.11=14.5 \%$, i.e. almost the same as the observed value of $14 \%$. In contrast, a model with a normal cloud metallicity predicts a flux dip of about $22 \%$ for $\lambda \lambda 1800-1900$, which may be excluded easily by the observations. Altogether, the metallic abundance of the cloud in $\sigma$ Ori E derived from a comparison of flux amplitudes in the middle- and near-UV is in very good agreement with our line synthesis analysis (Sect. 4.1). It is also in agreement with our general picture which requires that the chemical composition of the cloud should be essentially identical with the source material from the outflowing regions of the photosphere. 

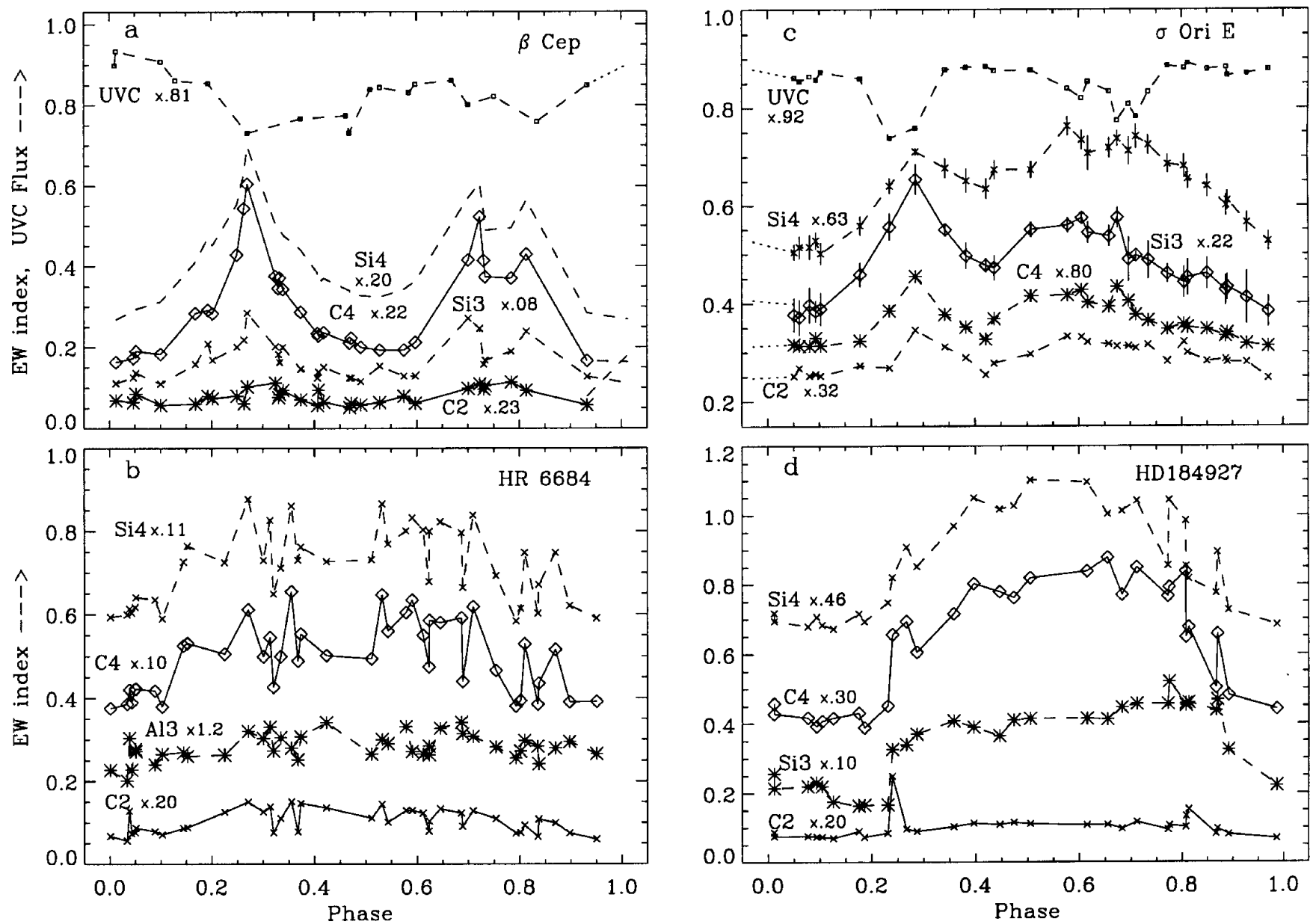

Fig. 4. a-d) Equivalent width indices of various lines of the program stars with rotational phase (periods given in text). In each case the index is defined as the reciprocal of the ratio of the fluxes within a $\Delta \lambda$ bandpass around line center (Table 2) to the ratio of valid fluxes outside these limits. The curves given are for Si IV $\lambda 1394,1403$ (mean), C IV $\lambda 1548$, Si III $\lambda 1206$, C II $\lambda 1298$. The curves are renormalized by the factors shown in the annotations and separated by vertical shifts.

\subsection{Cloud parameters from weak resonance lines}

In this and the next subsection we consider the formation of the added absorptions of weak and/or strong resonance lines of silicon, carbon, and nitrogen by using CIRCUS models to match the added absorptions observed at occultation phases. We will also discuss the necessary roles of turbulence and non-LTE processes for strong resonance lines formed in the cloud.

Table 2 gives the extreme equivalent widths in Angströms observed at clear-star and occultation phases. These strengths are measured with wavelength limits specified around line center (generally roughly given by $\pm v \sin i$ ) in order to isolate spectroscopic variations of a cloud from other circumstellar activity, such as the wind. The technique of measuring line strength variations in specific wavelength bands of $I U E$ spectra accurately was introduced by Shore \& Adelman (1981) for the He-strong stars. To gain some perspective of how representative the variations are in the program stars, we also measured the equivalent widths of the same lines in the archival datasets of other He-strong stars studied by SB90 with known periods. We found that the range of variations for $\sigma$ Ori $\mathrm{E}$ is typical for this subclass. For example, the range for the Si IV doublet of $\sigma$ Ori $\mathrm{E}$ is similar to that found for HD 37017, larger than for HD 37776, and smaller than for HD 64760.

For the weak resonance lines the differences between the observed equivalent widths in Table 2, in parentheses, is taken to be the total absorption contribution in the star's cloud(s). This amount can be compared directly with the detailed absorption predictions of $C I R C U S$, given in Table 3 for $\beta$ Cep and HD 184927. For a stated variation of a given line, the star-to-star differences can arise from a variety of factors, but the dominant one is the star's metallicity. Thus, similar cloud absorptions for lines of the similarly metal-deficient stars HD 184927 and HR 6684 will lead to similar derived cloud parameters. We also note that the dependence of the derived cloud parameters on metallicity is especially important for the strong resonance lines for which the greatest contrast exists between a (weak) photospheric line and a highly saturated cloud line. 
Table 2. Observed equivalent width extrema, Notes: (1) Units of equivalent-width $(E W \mathrm{~s})$ and wavelengths are Angströms were measured for $\Delta \lambda$ range noted. (2) Parenthesized entries after Min are differences between maximum and minimum $E W \mathrm{~s}$ at midpoints of clear-star and occultation phases; a colon denotes an uncertain value. (3) Bracketed entries following the listed ion is the ionization potential in eV. (4) Blank entries for Si IV and N V indicate that models are not computed for these ions in Table 3. (5) N V entries (starred) for HD 184927 and $\beta$ Cep refer to full-profile absorptions and are modeled in Table 3.

\begin{tabular}{|c|c|c|c|c|c|c|c|c|c|c|c|c|}
\hline & \multicolumn{3}{|c|}{$\sigma$ Ori E } & \multicolumn{3}{|c|}{ HD 184927} & \multicolumn{3}{|c|}{$\beta$ Сер } & \multicolumn{3}{|c|}{ HR 6684} \\
\hline Ion $[\mathrm{eV}]$ & $E W$ & $E W$ & $\Delta \lambda$ & $E W$ & $E W$ & $\Delta \lambda$ & $E W$ & $E W$ & $\Delta \lambda$ & $E W$ & $E W$ & $\Delta \lambda$ \\
\hline Line & Max & Min & $(\AA)$ & Max & Min & $(\AA)$ & Max & Min & $(\AA)$ & Max & Min & $(\AA)$ \\
\hline C II [24.4] & & & & & & & & & & & & \\
\hline 1336 & 682: & $.557:(.125)$ & 1.3 & $.520:$ & $.370:(.150)$ & 0.5 & $.292:$ & $.245:(.047)$ & 0.6 & 1.00: & $.880:(.120)$ & 1.1 \\
\hline C IV [64.5] & & & & & & & & & & & & \\
\hline 1548 & .944 & $.545(-)$ & 1.5 & .838 & $.158(-)$ & 1.3 & .890 & $.483(-)$ & 1.1 & 1.085 & $.234(-)$ & 1.2 \\
\hline 1550 & .794 & $.448(-)$ & 1.5 & .838 & $.158(-)$ & 1.3 & .890 & $.483(-)$ & 1.1 & .863 & $.157(-)$ & 1.2 \\
\hline N V [97.9] & & & & & & & & & & & & \\
\hline 1238 & .192 & $.042(-)$ & 1.5 & .421 & $.158(.530)^{*}$ & 1.3 & .506 & $-.097(.750)^{*}$ & 1.1 & .303 & $-.035(-)$ & 1.2 \\
\hline$\overline{\mathrm{Si} I I}$ [16.3] & & & & & & & & & & & & \\
\hline 1265 & .198 & $.150(.048)$ & 1.0 & .385 & $.372(.013)$ & 1.0 & .245 & $.248(.00)$ & 1.1 & .171 & $.164(.007)$ & 1.2 \\
\hline 1309 & .122 & $.074(.048)$ & 1.0 & .356 & $.316(.040)$ & 1.3 & .090 & $.071(.019)$ & 1.1 & .288 & $.268(.020)$ & 1.2 \\
\hline Si III [33.5] & & & & & & & & & & & & \\
\hline 1206 & .614 & $.395(.219)$ & 1.4 & .990 & $.739(.251)$ & 1.0 & .778 & $.435(.343)$ & 1.2 & 1.661 & $1.315(.346)$ & 1.2 \\
\hline 1301 & .247 & $.191(.056)$ & 1.5 & .416 & $.368(.048)$ & 0.6 & .346 & $.342(.004)$ & 0.6 & .417 & $.397(.020)$ & 0.8 \\
\hline 1303 & .227 & $.167(.060)$ & 1.5 & .435 & $.399(.036)$ & 0.6 & .316 & $.311(.005)$ & 0.6 & .402 & $.392(.010)$ & 0.8 \\
\hline 1892 & .459 & $.339(.120)$ & 1.5 & .479 & $.416(.063)$ & 0.6 & .524 & $.467(.057)$ & 0.6 & .406 & $.394(.012)$ & 0.8 \\
\hline 1895 & .353 & $.198(.155)$ & 1.5 & .476 & $.424(.052)$ & 0.6 & .460 & $.407(.053)$ & 0.6 & .376 & $.364(.012)$ & 0.8 \\
\hline Si IV [45.1] & & & & & & & & & & & & \\
\hline 1394 & .810 & $.589(.221)$ & 1.4 & .626 & $.377(.249)$ & 1.2 & .778 & $.542(.236)$ & 1.0 & 1.207 & $.817(.390)$ & 1.4 \\
\hline 1403 & .662 & $.426(.236)$ & 1.4 & .471 & $.258(.213)$ & 1.2 & .770 & $.523(.247)$ & 1.0 & .871 & $.711(.160)$ & 1.4 \\
\hline $\mathrm{Al} \mathrm{III}[28.4]$ & & & & & & & & & & & & \\
\hline 1855 & .551 & $.322(.229)$ & 1.6 & .633 & $.319(.314)$ & 0.8 & .772 & $.449(.323)$ & 1.3 & .629 & $.353(.276)$ & 1.4 \\
\hline 1862 & .480 & $.260(.120)$ & 1.6 & .434 & $.302(.132)$ & 0.8 & .434 & $.320(.114)$ & 1.3 & .556 & $.270(.286)$ & 1.4 \\
\hline
\end{tabular}

\subsubsection{CII, Sill and weak Silll lines}

In Table 4 we summarize our findings from Tables 2 and 3 by giving temperatures derived from C II, Si II, weak Si III, and $\mathrm{NV}$ absorptions. We kept microturbulence constant $\left(20 \mathrm{~km} \mathrm{~s}^{-1}\right.$, except for $\left.\mathrm{N} \mathrm{V}\right)$ in this compilation and took entries from the middle of the limits given in Table 3 . Before consolidating these results, we discuss the status of the suitability of these lines in terms of possible practical problems.

The C II $\lambda \lambda 1335-6$ doublet is a well-known temperature diagnostic for cloud-like conditions, but in IUE spectra the $1335 \AA$ line is marred by the presence of an instrumental reseau. In the spectra of $\sigma$ Ori E the broadened wings of the $1336 \mathrm{~A}$ feature are blended by nearby low excitation Cr II and Fe II photospheric lines, rendering $\lambda 1336$ unusable for this star. Both lines are further marred by an interstellar component (particularly for $\sigma$ Ori E). Despite these problems the C II lines noticeably broaden in occultation phase spectra of all our stars. Reference to Table 2 shows that the $\lambda 1336$ variation is moderately large for HD 184927 and HR 6684. Indeed, these are the only C II results that can be quoted with reasonable reliability.

$I U E / \mathrm{SWP}$ spectra also include the Si II resonance lines at $1264.7 \AA, 1265.0 \AA$, and $1309.3 \AA$. The observed variations for the doublet are immeasurably small for all the program stars except $\sigma$ Ori E. For $\beta$ Cep, HD 184927, and
HR 6684 the small variations mean that the $\mathrm{Si}^{1+}$ population is low because the cloud temperature is high (or, less likely, very low).

Variations in both weak resonance and low-excitation Si III lines provide convenient cloud temperature diagnostics. Consider first the forbidden Si III $\lambda 1892$ resonance line, for which the $\log g f$ is 30000 times weaker than the $\lambda 1206$ line's. This line is weakly visible in spectra of normal early B-type stars (Walborn et al. 1995), but it strengthens for our program stars during occultation phases. This is not surprising: in contrast to the photosphere, one expects a cool circumstellar gas with a long path length and a shallow temperature gradient to produce a strong absorption in a resonance line.

The Si III lines also shed light on the cloud density. Consider that the $\lambda 1892$ resonance line in turn feeds the excited lower levels of the $\lambda \lambda 1294-1303$ multiplet of Si III. Both the observations (Table 2) and the CIRCUS models demonstrate that the variations of the Si III resonance and multiplet features are comparable and well correlated among themselves. These facts suggest that metastability of the ${ }^{3} \mathrm{P}^{\circ} 3 \mathrm{p}$ (lower) level does not determine its atomic population and thus that the cloud densities are moderately high $\left(\geq 10^{11} \mathrm{~cm}^{-3}\right)$.

To summarize this section, the ionization potentials of $\mathrm{Si}^{1+}, \mathrm{C}^{1+}$, and $\mathrm{Si}^{2+}$ (SiII, CII, Si III: $\chi_{\mathrm{IP}}=16-34 \mathrm{eV}$ ) together provide an excellent bridge of diagnostics for 
Table 3. Computed cloud absorptions. Notes: (1) Cloud temperatures are given in Kelvins, and microturbulences in km $\mathrm{s}^{-1}$. (2) Columns (CD1-3) denote column density (units of $10^{22} \mathrm{~cm}^{-2}$ ) for models. (3) Note that the absorption entries are computed from the same $\Delta \lambda$ limits (except for N V) as given in Table 2. (4) Pairs of entries close to observed values in Table 2 are italicized. (5) Computed $E W$ s are often somewhat below or above the observed value. In such cases the table entries have trailing $(-)$ or preceding $(+)$ symbols, respectively. All models have coverage factors of $100 \%$.

\begin{tabular}{|c|c|c|c|c|c|c|c|c|c|c|}
\hline & HD 18492 & & & $E W$ & & $\beta$ Cep & & & $E W$ & \\
\hline $\begin{array}{c}\text { Ion/Line } \\
\text { C II }\end{array}$ & $T_{\text {cloud }}$ & $v_{\text {turb }}$ & $\begin{array}{c}\text { CD1 } \\
1 \\
\end{array}$ & $\begin{array}{c}\text { CD2 } \\
3.2 \\
\end{array}$ & $\begin{array}{c}\text { CD3 } \\
10 \\
\end{array}$ & $T_{\text {cloud }}$ & $v_{\text {turb }}$ & $\begin{array}{c}\text { CD1 } \\
1 \\
\end{array}$ & $\begin{array}{c}\text { CD2 } \\
3.2 \\
\end{array}$ & $\begin{array}{c}\text { CD3 } \\
10 \\
\end{array}$ \\
\hline $1336 \AA$ & $\begin{array}{l}15000 \\
14000 \\
13000 \\
\end{array}$ & $\begin{array}{l}20 \\
20 \\
20 \\
\end{array}$ & $\begin{array}{l}.121 \\
.147 \\
\end{array}$ & $\begin{array}{l}.118 \\
.151 \\
.169 \\
\end{array}$ & $\begin{array}{l}.146 \\
.178\end{array}$ & $\begin{array}{l}17000 \\
16000 \\
15000 \\
\end{array}$ & $\begin{array}{l}20 \\
20 \\
20 \\
\end{array}$ & $\begin{array}{r}.124 \\
.152 \\
\end{array}$ & $\begin{array}{l}.119 \\
.150 \\
.182 \\
\end{array}$ & $\begin{array}{l}.146 \\
.173 \\
.199 \\
\end{array}$ \\
\hline N V & & & .32 & 1 & 3.2 & & & .32 & 1 & 3.2 \\
\hline $1238 \AA$ & $\begin{array}{l}31000 \\
30000\end{array}$ & $\begin{array}{l}50 \\
50\end{array}$ & $.252-$ & $\begin{array}{l}.478- \\
.282\end{array}$ & $\begin{array}{c}+.589 \\
.511\end{array}$ & $\begin{array}{l}29000 \\
28000 \\
28000 \\
\end{array}$ & $\begin{array}{c}50 \\
50 \\
100 \\
\end{array}$ & & $\begin{array}{l}.742 \\
.585 \\
.742 \\
\end{array}$ & \\
\hline Si II & & & 1 & 3.2 & 10 & & & 1 & 3.2 & 10 \\
\hline $1265 \AA$ & $\begin{array}{l}16000 \\
15000 \\
14000\end{array}$ & $\begin{array}{l}20 \\
20 \\
20\end{array}$ & $\begin{array}{l}.005- \\
.017\end{array}$ & $\begin{array}{r}.006- \\
+.020\end{array}$ & $\begin{array}{c}+.017 \\
.048\end{array}$ & $\begin{array}{c}\text { not } \\
\text { modeled }\end{array}$ & & & & \\
\hline $1303 \AA$ & $\begin{array}{l}15000 \\
14000 \\
13000 \\
\end{array}$ & $\begin{array}{l}20 \\
20 \\
20 \\
\end{array}$ & $\begin{array}{l}.024- \\
.062 \\
\end{array}$ & $\begin{array}{l}.026- \\
+.068\end{array}$ & +.068 & $\begin{array}{l}18000 \\
17000 \\
16000 \\
\end{array}$ & $\begin{array}{l}20 \\
20 \\
20 \\
\end{array}$ & $.012-$ & $\begin{array}{r}.009- \\
+.035 \\
\end{array}$ & $\begin{array}{r}.020 \\
+.030 \\
.075 \\
\end{array}$ \\
\hline Si III & & & 1 & 3.2 & 10 & & & 1 & 3.2 & 10 \\
\hline $1206 \AA$ & 16000 & 50 & & $.216-$ & +.260 & $\begin{array}{l}17000 \\
17000 \\
16000 \\
16000 \\
15000 \\
15000\end{array}$ & $\begin{array}{l}20 \\
35 \\
20 \\
35 \\
20 \\
30\end{array}$ & $\begin{array}{l}.248 \\
.345 \\
.334\end{array}$ & $\begin{array}{l}.330 \\
.348 \\
.248- \\
.492\end{array}$ & $\begin{array}{r}.259 \\
\\
+.368\end{array}$ \\
\hline $1301 \AA$ & $\begin{array}{l}17000 \\
16000 \\
15000\end{array}$ & $\begin{array}{l}20 \\
20 \\
20\end{array}$ & $\begin{array}{l}.029- \\
.042 \\
.044\end{array}$ & +.048 & & $\begin{array}{l}19000 \\
18000 \\
17000 \\
16000\end{array}$ & $\begin{array}{l}20 \\
20 \\
20 \\
20\end{array}$ & $\begin{array}{l}.004 \\
.007\end{array}$ & $\begin{array}{l}.003- \\
(.03)\end{array}$ & $\begin{array}{r}.006 \\
+.009 \\
(.12)\end{array}$ \\
\hline $1892 \AA$ & $\begin{array}{l}16000 \\
15000 \\
14000\end{array}$ & $\begin{array}{l}20 \\
20 \\
20\end{array}$ & & $\begin{array}{l}.032- \\
.040- \\
.038-\end{array}$ & $\begin{array}{l}+.084 \\
+.097 \\
+.092\end{array}$ & $\begin{array}{l}20000 \\
19000 \\
18000 \\
17000\end{array}$ & $\begin{array}{l}20 \\
20 \\
20 \\
20 \\
\end{array}$ & $\begin{array}{l}.032- \\
.063\end{array}$ & $\begin{array}{l}.041- \\
+.079\end{array}$ & $\begin{array}{c}.052 \\
+.096\end{array}$ \\
\hline $\mathrm{Al} \mathrm{III}$ & & & 1 & 3.2 & 10 & & & 1 & 3.2 & 10 \\
\hline $1855 \AA$ & $\begin{array}{l}18000 \\
17000 \\
17000 \\
16000 \\
16000\end{array}$ & $\begin{array}{l}50 \\
20 \\
50 \\
20 \\
50\end{array}$ & $\begin{array}{l}.177 \\
.253- \\
.386\end{array}$ & $\begin{array}{c}.260- \\
+.432 \\
.241\end{array}$ & $\begin{array}{c}+.382 \\
.246 \\
.312\end{array}$ & $\begin{array}{l}17000 \\
16000 \\
16000 \\
15000 \\
15000\end{array}$ & $\begin{array}{l}50 \\
20 \\
50 \\
20 \\
50\end{array}$ & $\begin{array}{l}.278 \\
.315- \\
.301-\end{array}$ & $\begin{array}{c}.330 \\
.258 \\
+.340 \\
.281 \\
+.349\end{array}$ & $\begin{array}{l}.321 \\
.319\end{array}$ \\
\hline
\end{tabular}

conditions between the inner and outer cloud regions. From Table 4 one can see that the line-formation temperatures decrease with ionization potential of the parent ion, as one would expect for a circumstellar cloud having an outward-decreasing temperature.

\subsection{Resonance line absorptions as diagnostics}

\subsubsection{Probing temperature and geometry}

According to our CIRCUS models, the ionization of silicon and carbon most favors line formations at temperatures 
Table 4. Synopsis of best-fit cloud temperatures for lines of ions listed (from Table 3). Notes: (1) Typical column densities are in middle of best-fit parameter space. (2) Errors are typically $\pm 1000 \mathrm{~K}$ except for C II(ISM and reseau flaws) and Si III ( $\beta$ Cep). (3) Typical microturbulence values are $20 \mathrm{~km} \mathrm{~s}^{-1}$, except for NV $\left(50 \mathrm{~km} \mathrm{~s}^{-1}\right)$.

\begin{tabular}{c|c|c|c}
\hline \hline Line & $\begin{array}{c}\text { column } \\
\text { density }\end{array}$ & HD 184927 & $\beta$ Cep \\
\hline & & & \\
C II & $110^{23}$ & $15000 \mathrm{~K}$ & (none) \\
Si II & $310^{22}$ & $15000 \mathrm{~K}$ & $16500 \mathrm{~K}$ \\
Si III & $310^{22}$ & $16500 \mathrm{~K}$ & $18500 \mathrm{~K}$ \\
N V & $110^{22}$ & $30500 \mathrm{~K}$ & $28500 \mathrm{~K}$ \\
\hline
\end{tabular}

centered at $21000 \mathrm{~K}$ and $26000 \mathrm{~K}$, respectively, for the expected cloud densities. These temperatures are comparable and slightly higher, respectively, than what one might expect from radiative equilibrium models for plasma near stars having $T_{\text {eff's }}$ of $23000-27000 \mathrm{~K}$. Thus although the resonance lines are not necessarily superionized features, they must be formed in a warm environment, thus close to the star. As noted by various authors (Fischel \& Sparks 1980; Barker et al. 1982; Shore \& Brown 1990; Henrichs et al. 1998), the strong resonance lines of C IV, Si IV and other ions undergo dramatic changes during the rotational cycle of each of our program stars. Figure 4 depicts the phase variations of an equivalent width index for several strong resonance lines for each program star ${ }^{4}$. In each case this index is the ratio of the measured fluxes outside and inside the $\Delta \lambda$ wavelength limits for that line, as specified in Table 2. Similar indices computed from the blue and red halves of these wavelength windows give in all cases essentially the same fractional amplitudes. These checks indicate that the influences of the wind at moderate velocities (or in the case of the CIV lines, the Fe III interstellar components) do not contribute appreciably to the variation of this index. The sequence of these strong line variations may also be compared qualitatively to the variations of the Al III resonance doublet by consulting Fig. 1.

The data in Fig. 4 are well enough sampled to draw several conclusions concerning the geometry of the tori. First, the absorptions among different strong resonance lines peak at the same small ranges of phase. This fact suggests that these lines form over the same stellar longitude. However, for $\beta \mathrm{Cep}$ and $\sigma$ Ori E the ultraviolet quasicontinuum curves are offset from the central absorption phases of the strong resonance lines. This fact implies a warping at large distances from the star (see also Groote \& Hunger 1997, GH97). Another conclusion from the sharpness of the maxima in these figures is that the heights of the clouds (perpendicular to their plane of symmetry) are

${ }^{4}$ The depicted UVC curves for $\beta$ Cep and $\sigma$ Ori $\mathrm{E}$ are time sequences of binned fluxes constructed from the echelle orders extending over $\lambda \lambda 1800-1905$; errors are estimated to be $\pm 3 \%$. The $\beta$ Cep UVC data are large-aperture observations of 1991-4 and are corrected for a $5 \%$ sinusoid found in the raw data at the pulsation period. The $\sigma$ Ori E UVC fluxes are from 1980-1. at least one stellar diameter. Otherwise, for short heights, their optically thick absorptions would be proportional to their projected areas against the stellar disk. Such a geometry would impose an (unobserved) local minimum on the resonance-line absorption curve at the phase of mid-occultation. We used this fact to adopt an area coverage factor of $100 \%$ in our CIRCUS analysis of cloud parameters.

\subsubsection{Probing the wind}

To investigate the behavior of the blue-wing absorption from winds in these stars, we modified our equivalent width extraction routine to compute flux ratios in a $2 \AA$ segment of this wing to all valid fluxes in the order except those from the photospheric line profile. With the contribution of the latter suppressed, we found no variation (i.e., to $<10 \%$ ) in the wings of either the SiIV or C IV lines with rotation phase among any of the program stars. Additionally, the blue edge velocities are stable over the cycle and are also remarkably uniform from star to star. GH97 had already found that the coronal wind in $\sigma$ Ori $\mathrm{E}$ is phase-independent, and the constancy we find tends to extend this conclusion to our other stars. This is a notable point because previous investigators have stated that the wind fluxes of HD 184927 vary from this star's magnetic pole to equator (Vauclair et al. 1991). This erroneous perception has been an important supporting tenet for the model of photospheric diffusive settling of helium (Michaud et al. 1987). In addition, these observations suggest that the initial wind column, dubbed the jet by SB90, is not visible in these data, though indeed it must ultimately be visible at some level of photometric precision because of the optical thinness in the far blue wings of the photospheric lines. The difficulty of observing the jets could mean that their velocities are spread out over a large range.

\subsubsection{Probing density and turbulence}

Figure 5 depicts the total ranges in absorption and emission from pairs of observations for the strong resonance lines for the program stars. Inspection of these figures shows that profiles at clear-star (dashed lines) phases differ from occultation phase profiles (solid lines) in the following ways: (1) the clear-star profiles of C IV and Si IV exhibit emissions in their cores and red wings (Sect. 5), (2) the Si III profiles exhibit narrower cores, and (3) the red and blue far-wings of these lines are strong relative to photospheric line models.

As with the weaker metallic lines, one would hope to utilize the strengths of strong resonance lines at clearstar phases as baselines to compare with the absorptions at occultation phases. However, modeling the strong resonance lines is a problem complicated by the presence of noncoherent scattering (partial redistribution) in the line source function as well as illumination of the torus 


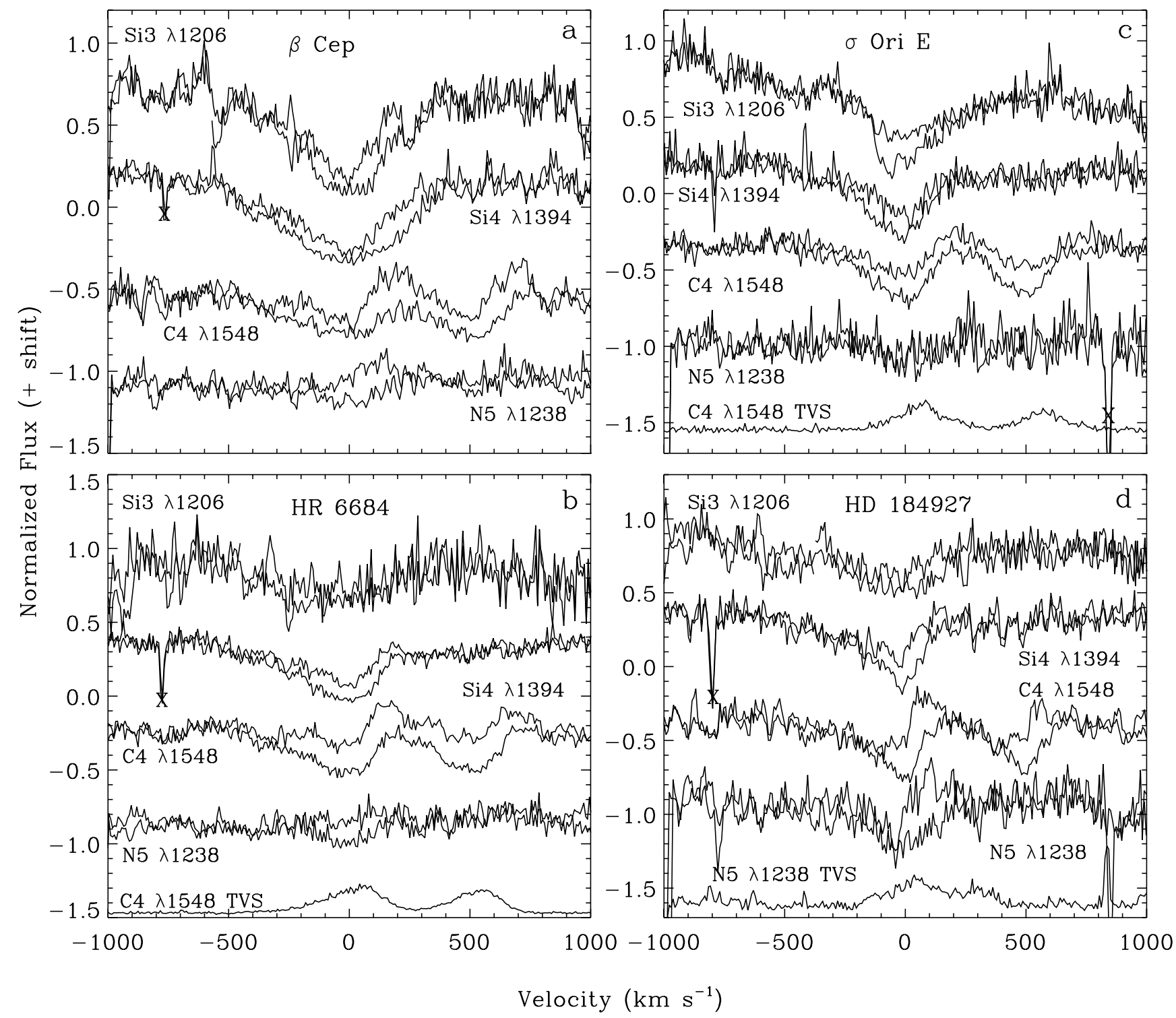

Fig. 5. a-d) Comparison of spectra of Si III, Si IV, CIV, and N V resonance lines of minimum (clear-star phase; dashed line) and maximum (occultation phase, solid line) absorption strength (shifted for clarity). Except for $\beta$ Cep the normalized TVS spectrum is also given. a) For $\beta$ Cep, the corresponding spectra are from SWP 57573 (dashed line) and SWP 52514 (solid line), b) for HR 6684 the corresponding spectra are from SWP 55732 and SWP 55526 , c) for $\sigma$ Ori E the corresponding spectra are from SWP 07560 and occultation phase SWP 15787, d) corresponding spectra for HD 184927 are from SWP 55652 and SWP 55685.

by a diffuse radiation source (the jet). In general, it is difficult to separate the effects of the torus from both the photosphere and a jet. In particular, it is impossible in practice to identify a reference photospheric profile from which to difference absorption and emission of the toruscloud because the observed Si IV, C IV, and N V line profiles are peculiar at all phases, exhibiting strong absorptions during occultations and emissions at other times. Nonetheless, points 2 and 3 above can provide hints of local cloud conditions. For example, point \#2 suggests that the broad core of the occultation-phase profiles is a signature of velocity broadening. As to point \#3, the extended, particularly red wings of the Si III and Si IV lines can best be understood as enhanced strengthenings from a dense cloud. Consider that our CIRCUS models for Si III $\lambda 1206$ and the Si IV resonance doublet indicate that densities less than $10^{11} \mathrm{~cm}^{-3}$ cannot be distinguished from the clearstar (photospheric) profiles. Our best models require cloud densities in the range of $10^{12-13} \mathrm{~cm}^{-3}$. Taking again the simplest paradigm that the cloud temperature increases inward toward the star, the wings of the strong silicon resonance lines suggest then that cloud densities increase inwards too.

Traces of the variations so readily apparent in the Si IV, CIV, and N V lines are still present in Si III $\lambda 1206$ and even in the AlIII doublet. However, the difference 
between this line and the others is that the Si III line does not show any emission at any phase. While this circumstance might lead to the hope that one can model the $\lambda 1206$ variations, we found in practice that we could not reproduce the observed strengthening of this line without introducing a substantial microturbulence. As an example, consider the Si III line in $\beta$ Cep. Table 2 shows a cloud-added absorption of $343 \mathrm{~m} \AA$. CIRCUS models suggest a maximum absorption for $T_{\text {cloud }}=$ $17000-19000 \mathrm{~K}$ (Sect. 4.4.1). Even for column densities of $3-10 \times 10^{22} \mathrm{~cm}^{-2}$ and a turbulence of $20 \mathrm{~km} \mathrm{~s}^{-1}$, our simulations predict an absorption in the range 138-259 $\mathrm{m} \AA$. Low-temperature, pure-absorption (non-LTE) models also cannot reproduce the observed strengthening. If one introduces a high turbulence of $50 \mathrm{~km} \mathrm{~s}^{-1}$ and also adopts a reasonable temperature and column density $(17000 \mathrm{~K}$ and $\left.3 \times 10^{22} \mathrm{~cm}^{-2}\right)$, the observed absorptions $(\approx 340 \mathrm{~m} \AA)$, can then be reproduced. The Si III line's sensitivity to turbulence implied here is a consequence of the saturation in the core. One may assume that the broad cores of the C IV and Si IV lines at occultation phases are also due to turbulence. As for Si III $\lambda 1206$, moderately high turbulences and moderate temperatures produce good fits to the Al III doublet absorptions, noted in Table 3 . We also note similar good fits for HD 184927. This is not surprising in view of the similar ionization potential of this ion: the Al III lines are probably formed in nearly the same region of the cloud as Si III $\lambda 1206$.

\subsubsection{The $\mathrm{NV}$ absorptions}

The anomalously strong absorptions of the N V $1238 \AA$, $1242 \AA$ doublet at occultation phases first drew our attention to the rotating magnetic B stars. According to stellar atlases (e.g. Walborn et al. 1995), the spectra of normal B0-B2 V stars show no trace of this doublet. Even for the hot star $10 \mathrm{Lac}\left(\mathrm{O} 9 ; T_{\text {eff }}=35400 \mathrm{~K}\right)$, we estimate that this line has a photospheric strength of only $175 \mathrm{~m} \AA$ (see the Brandt et al. 1998 atlas). Our SYNSPEC models reveal that their presence is difficult to discern in photospheric spectra in stars with $T_{\text {eff's }}$ below about $28000 \mathrm{~K}$ because of nearby line blends. Grigsby \& Morrison (1995) have recently noted the anomalous strengths of $\mathrm{N} V$ lines in spectra of the so-called nitrogen enhanced late-O stars (Walborn et al. 1985). They attribute them to a photoionization of the wind by X-rays. However, the wind explanation cannot apply to the rotating magnetic stars in our sample because the lines do not show extended, high-velocity (blue) wings. Moreover, the NV strengths in our program stars tend to be larger than the features in these O-stars. By elimination, we conclude that the $\mathrm{N} \mathrm{V}$ absorptions are formed in the circumstellar cloud(s).

The NV absorptions in these stars are so large $(\approx 750 \mathrm{~m} \AA$ and $\approx 530 \mathrm{~m} \AA$ over the full profile for $\beta$ Cep and HD 184927, respectively) that they cannot be caused by enhanced abundances or strong winds, or fit by our conventional strategies. For example, our standard cloud

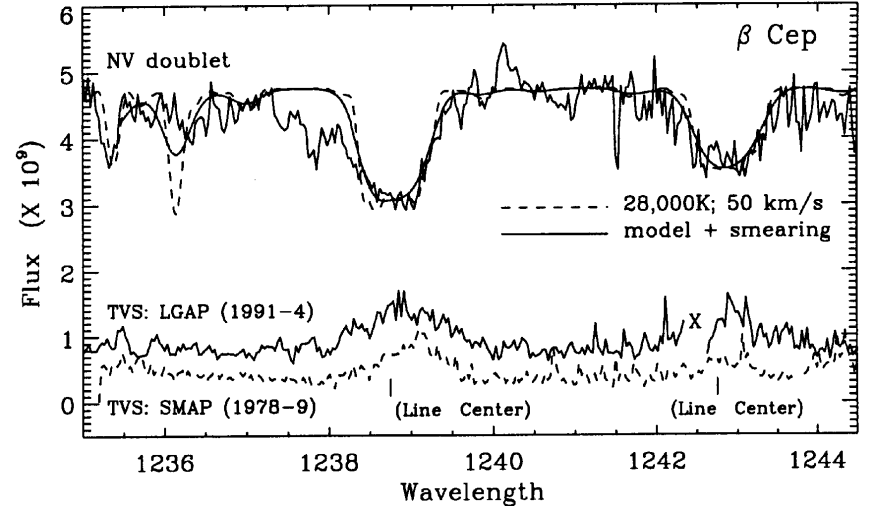

Fig. 6. Comparison of mean spectrum of the N V doublet for $\beta$ Cep compared to a CIRCUS model fit (upper panel). The observations are taken from the the occultation-phase spectra listed in Fig. 2c. The fitted model represents a cloud with the temperature and microturbulence shown. The dashed fit is the result of smoothing with a $100 \mathrm{~km} \mathrm{~s}^{-1}$ Gaussian. Lower panel shows the TVS spectra divided by mean spectra for the ensemble of large- (LGAP) and small-aperture (SMAP) observations of $\beta$ Cep.

models, with both high cloud temperatures $(\sim 33000 \mathrm{~K})$ and a very large high column density $\left(10^{23} \mathrm{~cm}^{-2}\right)$ can not reproduce them. It is only when one considers models with both high cloud temperatures and microturbulences together that the strong absorptions can be matched. For such combinations the precise strength of the absorption is insensitive to column length because the line is already so opaque. To assess the effects of increasing both these parameters, we carefully computed the underlying photospheric spectrum, taking into account the star's $T_{\text {eff }}$, $\log g$, and $v \sin i$. The CIRCUS models showed that the $\mathrm{N}$ V line is extremely sensitive to temperature, a fact that greatly restricts the range of variables in successful models. For $\beta$ Cep we settled on a promising pair of models (normal abundances, column density of $10^{22} \mathrm{~cm}^{-2}$, microturbulence $=50 \mathrm{kms}^{-1}$ ). Then, a change from $28000 \mathrm{~K}$ to $29000 \mathrm{~K}$ strengthens the equivalent width from 585 to $742 \mathrm{~mA}$. Increasing the microturbulence from 50 to $100 \mathrm{kms}^{-1}$ produces exactly the same increase. Thus, both microturbulence and temperature are tightly constrained by the strengthening of this line. We obtained similar temperatures for $\sigma$ OriE and HR 6684, with a turbulence of $50 \mathrm{~km} \mathrm{~s}^{-1}$. For HD 184927 we were able to match the absorption with a temperature of $30000-$ $31000 \mathrm{~K}$, assuming a column density of $1-2 \times 10^{22} \mathrm{~cm}^{-2}$ and $50 \mathrm{kms}^{-1}$. The best fit models are summarized in Table 4.

Figure 6 exhibits a model fit of the N V doublet for $\beta$ Cep at the occultation phase. The fitted cloud absorption profile was computed with a CIRCUS model having a coverage factor of $100 \%$, heated $T_{\text {cloud }}=29000 \mathrm{~K}$, a column density of $3.2 \times 10^{22} \mathrm{~cm}^{-2}$, a microturbulence of $50 \mathrm{kms}^{-1}$, and a further convolution with a $100 \mathrm{~km} \mathrm{~s}^{-1}$ Gaussian. This macroturbulence-smearing was necessary to remove the corners of a boxy-shape profile produced 
by microturbulence alone. The parameters required to approximate the N V absorption of HD 184927 are similar. Because HD 184927 has a low surface metallicity, the required cloud temperature is a little higher, $\approx 31000 \mathrm{~K}$, and the derived micro- and macro-turbulences are then $50 \mathrm{~km} \mathrm{~s}^{-1}$ and $100 \mathrm{~km} \mathrm{~s}^{-1}$, respectively.

\section{Resonance line emissions}

The spectroscopic hallmark of the rotating magnetic $B$ stars is the strong variation of their C IV and N V doublet lines with rotational phase, ranging from strong absorption to redshifted emission. Since these variations are not necessarily well ordered, only a few generalizations can be made about their behavior. One overriding general characteristic is that the emission and/or absorption components are always present to some degree. A second characteristic is that although small differences can arise in samephase profiles of different epochs, the general variations are remarkably repeatable over at least several years. For example, a narrow redshifted emission feature seems to be a common feature in the $1238 \AA$ profiles of HD 184927 at phases near 0.85 and 0.15 , i.e. at the beginning and end of the range of clear-star phases. For some stars, such as HR 6684, the N V profiles almost always show emission components, even when much of the profile is strongly in absorption. For other stars like $\sigma$ Ori E, the N V emissions are difficult to detect at all. The weakness of N V emissions in $\sigma$ Ori E, along with the weakness of Si IV emissions for all of the program stars, probably explains why these emissions have gone unreported for so long in studies of He-strong stars.

All four of our stars show detectable emissions in lines of at least two ions. In two of our four program stars, the $\mathrm{NV}$ lines exhibit the strongest emissions, while for HR 6684 or $\sigma$ Ori E it is the CIV emissions which are the strongest.

In some cases, the $\mathrm{NV}$ emissions of these stars are almost completely confined to the red side of the profile, and indeed in all cases the TVS is larger there. The lower portions of Figs. 5b-d and 6, depict the TVS spectrum divided by the mean profile for the CIV profiles or $\mathrm{NV}$ profiles. We depict the ratio of the TVS to the mean spectrum in order to account for absorptions of the strong line core which would otherwise suppress fluctuations at low velocities. The TVS-ratio profiles generally peak at $+60-125 \mathrm{~km} \mathrm{~s}^{-1}$, and their red wings extend to $+300-400 \mathrm{~km} \mathrm{~s}^{-1}$ in each star with remarkable uniformity. Thus, the edge velocities are not related to the peak emission strength, the star's metallicity, pulsational status, or $v \sin i$.

A comparison of the TVS-ratio spectrum for $\beta$ Cep among both large- and small-aperture observations in Fig. 6 gives a qualitative idea of the robustness and constancy of the activity spectrum over even widely separated epochs for this star. It is interesting that the TVS profiles for C IV and N V do not exhibit a tail extending to the far blue wing. This fact shows that the stars' winds do not vary much over stellar longitude. In all, one finds that the redshifted emissions are uncorrelated with wind visibility in the blue wings of the lines. Likewise, the emissions do not appear to arise from the wind, so one must look to a different mechanism to explain them too.

We can quantify the temperature range required to reproduce the $\mathrm{N}$ V emissions by again using CIRCUS models, again taking $\beta$ Cep as an example. As one increases the cloud plasma temperature in these models, the N V lines first show increased absorptions at temperatures. These absorptions peak in the range $\sim 35000-40000 \mathrm{~K}$, both for LTE and non-LTE models. As the temperature increases the line goes into emission. One may place interesting upper limits on the line formation region of the $\mathrm{N} \mathrm{V}$ emissions because at temperatures in excess of $40000 \mathrm{~K}$ these regions of the cloud would contribute an unacceptably large fraction to the combined continuum flux of the star and cloud. Such models would produce a nearly sinuosoidal modulation in continuous flux as the torus co-rotates around the star. This is not indicated by our continuum light curve data. so we can conclude that any continuum flux from a bright emission patch near the poles is negligible. An additional consequence of these arguments is that the N V line emissions are optically thin. This result is also supported by the high ratio (1.5-2) of the relative rms amplitudes of the two lines in Fig. 6 (the doublet components have a $g f$ ratio of 2 ).

In assuming the optical thinness condition and an areal coverage factor of unity, it becomes straightforward to model the $\lambda 1238$ emission equivalent width. Again taking $\beta$ Cep as an example, the observed emission over the full profile is $\approx-220 \mathrm{~m} \AA$. In the models there is only a small trade-off between gas temperature and column density, e.g., a choice of parameters $49000 \mathrm{~K}$ and $3 \times 10^{17} \mathrm{~cm}^{-2}$ or $50000 \mathrm{~K}$ and $10^{18} \mathrm{~cm}^{-2}$; the limiting condition for $\tau_{\lambda 1238}=1$. Because of the sensitivity of $\mathrm{NV}$ ionization with temperature, the results for HD 184927 (full profile equivalent width $\sim-0.055 \AA$ ) are similar to those for $\beta$ Cep: we find an optimal temperature of $45000 \mathrm{~K}$ for the resulting length of the $\mathrm{NV}$ emission region is $1 \times 10^{11} \mathrm{~cm}^{-3}$, the $\mathrm{NV}$-emitting region comes out to be prohibitively thin, only $\sim 100 \mathrm{~km}$. This is roughly $100 \times$ smaller than a scale height in the torus. The shock depth can be reconciled to the expected range of values if the assumed coverage factor for $\mathrm{NV}$ emission is $\leq 1 \%$ rather than the initially assumed value of $100 \%$.

\section{Interpretation}

A key result of the previous section is that the UV line absorptions of a rotating magnetic B star vary because of a cloud passing in front of the star. Thus, there is no need to invoke (nor evidence for) an inhomogeneous metallic composition on the surfaces of these stars. The observations also suggest that the mass in these clouds is nearly constant over time. To maintain a constant mass, the wind accreted by the cloud must somehow settle back to the star and/or escape through its outer point. Indeed, perhaps it is the settling of cloud material onto the surface 
that is responsible for the high densities we found in the inner cloud regions. As a consequence of this recirculation, the cloud has virtually the same metallic composition as the star's polar caps and indeed the rest of its surface.

The numerical analysis of line strength variations in Sect. 4 indicates that the cloud line components form in an extended environment of a column density of roughly $1 \times 10^{23} \mathrm{~cm}^{-2}$. In the detailed models of the cloud spectra of HD 184927 and $\beta$ Cep, the lines are formed over a range of temperatures consistent with the stellar photosphere's radiation field. Although there is no evidence for long-term changes in cloud conditions, there is a strong suggestion of small- and large-scale turbulence in the inner regions. The strong wings of the Si IV and Si III resonance lines argue for increasing cloud densities in these regions, which seem to approach photospheric values at the inner edge of the torus.

The redshifted emissions in the N V and C IV lines observed during the magnetic pole-on phases demonstrate that downflows occur in the vicinity of the rotating, magnetic B-stars. In our picture they are most likely to arise from shocks because of the rapid deceleration of the wind particles as they impact the pre-existing torus (cf. Babel \& Montmerle 1996). This produces a redshift during the phase when the magnetic pole is visible because the wind has followed the curved lines of force and is then moving away from the observer. However, additional possibilities are possible. One should also consider the possibility of a stalled wind which impacts the star's surface near its point of origin. (In principle, it is also possible that magnetohydrodynamical processes, or Kelvin-Helmholtz instabilities; see Shore et al. 1987, operate, but we see no need for this in view of of the potential of steady-state hydrodynamical iteractions alone.)

In examining the evidence, we have found two emission characteristics that cast doubt on whether the emissions are produced by reaccretion, and we review them in the following. We discovered the first of these characteristics by recalling that SB90 had drawn attention to red-shaded C IV emissions in two He-strong stars for which periods are not known, HD 96446 and HD 58260. SB90 also noted that these features cannot arise from spherically symmetric shells, i.e. they are not true P-Cygni profiles. The SB90 star HD 96446 is likely to meet our criteria for membership among the rotating magnetic B stars because it also shows N V line emission. In addition, its emissions are variable. The case of HD 58260 is particularly interesting in this analysis for two reasons. First, SB90 have pointed out that we probably observe this star at a pole-on aspect (the magnetic and rotational poles are nearly coincident), so its emissions do not vary. Second, this star's C IV line emissions are both optically thick and strong - indeed, it attains a level of $0.5 I_{\text {cont }}$. In attempting to model C IV emissions, we found that it was not possible to reproduce this emission strength within the context of our models. Even our best model parameters $\left(T_{\text {cloud }}=30000 \mathrm{~K}\right.$, turbulence $=50-100 \mathrm{~km} \mathrm{~s}^{-1}$, high column densities, coverage factor $=1$ ) produce C IV emissions of only $0.20 I_{\text {cont }}$. We also found that the efficiency for producing the emission peaks very sharply at $30000 \mathrm{~K}$, so areal coverage factors

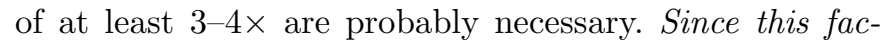
tor is significantly greater than one, the emissions cannot be produced just on the stellar surface. The area of the cloud-torus is more than adequate to produce the total emission, so we are led to the conclusion that the emissions are caused at the wind-cloud interface.

The second characteristic we used to decide between the competing shock models for line emission came from the detailed examination of the evolution of the C IV and $\mathrm{NV}$ emissions in both $\beta$ Cep and $\sigma$ Ori E. We discovered that an emission component appears first at the end of the occultation phase. In a model in which the downflows fall onto the star's surface, we would expect the line-emitting material to share the star's rotation because of its co-rotation with the star. As the cloud clears the receding limb of the star at the end of its occultation, emission is produced as the failed-wind stream shocks at a magnetic pole (now appearing at the opposite limb of the star). The emissions from this region will have an algebraically smaller radial velocity both because of the star's rotation and because the projected component of the infall velocity at this time is nearly zero. The emissions should then migrate redward smoothly across the line profile in this model as both velocities become more positive. The actual observations indicate that the emission component first appears weakly at a common point in the line profiles of both stars $\left(\sim-200 \mathrm{~km} \mathrm{~s}^{-1}\right)$ and then migrates to the red. This smooth behavior is suddenly interrupted by the strong appearance of a red emission component at $+0-100 \mathrm{~km} \mathrm{~s}^{-1}$. This particular evolution is difficult to understand as a consequence of shocks occurring on the stellar surface, particularly for $\beta$ Cep and $\sigma$ Ori E, which have very different rotational velocities. In contrast, the disk interpretation is almost too easy, suffering only from an embarrassment of several nonunique geometrical interpretations.

A related question is how the $\mathrm{NV}$ absorptions are formed during occultation phases, sometimes even coexisting with the emissions. The hypothesis that the $\mathrm{NV}$ emissions are emitted from the torus interface provides an advantage of restricting the formation of the emission to the same general site as the absorption. Both sites require a high temperature. Unlike the emission component, the absorption is optically thick. CIRCUS models show that absorption of $\mathrm{NV}$ is necessarily formed in a turbulent medium having a warm temperature of about $30000 \mathrm{~K}$. These results suggest that the NV absorption is formed in a large region of the cloud in which $\mathrm{CIV}$ and $\mathrm{NV}$ are overionized. We suggest that the wind-cloud shocks generate this overionization.

Still, it is not clear whether the heating required to produce the $\mathrm{NV}$ absorption occurs from a conversion of mechanical or radiative energy. The problem with either mechanism is that it is difficult to heat a large enough cloud volume to produce the amount of absorption observed. For example, turbulence can circulate 
shock-heated gas but probably only to a few scale heights. For representative cloud parameters $\left(30000 \mathrm{~K}, 10^{11} \mathrm{~cm}^{-3}\right)$ this length is probably only $10^{5} \mathrm{~km}$ or less. Radiative models encounter similar difficulties if the radiation from the shocks is emitted primarily in the EUV. The photoionization radius for $\mathrm{N}^{4+}$ in our cloud models is only $\sim 10^{3} \mathrm{~km}$. In contrast, CIRCUS models that match the N V absorptions require a full areal coverage factor, so the scale length for energy dissipation must be at least $1 R_{*}$. The radiative model can be saved if the shocks generate soft X-rays $(1 / 2-1 \mathrm{keV})$ which would be transparent to the cloud over its full length. Such irradiation would produce a broad distribution of ion states (Cohen et al. 1994) and would thus account for the observed strong absorptions produced by the cloud over a range of ion stages. The evidence for X-ray heating on these stars is ambiguous. Although the mean $\mathrm{X}$-ray levels are known to be low, little is known yet about variability. The kinetic model in turn might be reconciled with models of relativistic particles penetrating to great depths into the torus of $\sigma$ Ori E. Alternatively, it may be that porosity, rarefied zones, and/or Alfvén waves play roles in transporting mechanical energy into the cloud. Although we favor the radiative model, all of these possibilities are speculative. Thus, the question of the origin of the N V absorptions remains open.

\section{Conclusions}

The present work confirms the finding by Henrichs et al. (1998) that a separate class of rotating magnetic stars exists among the early-type main sequence B stars. Taking $\beta$ Cep as a case in point, one finds that a dipolar field of only a few hundred gauss can suffice to guide a polar wind from the surface to its equatorial regions via closed magnetic loops. The wind particles are abruptly halted near the magnetic equator where they establish a quasistatic, torus-shape structure (SB90). Our picture differs from the picture of GH97 and other investigators in two important respects. First, we have found that variations of the UV and probably optical lines (other than of helium) are due to absorptions in the torus-cloud. Second, we have remarked that the blue wings of the Si III, Si IV, and C IV resonance lines remain remarkably constant with rotational phase for all the program stars. Let us examine a few ramifications of these results.

If indeed the variable line absorptions are caused by the clouds, and if the metallic composition is nearly constant across their surfaces, their low-metallicities all over the surface will be maintained only if cloud material settles back to the star more efficiently than its wind streams upward. One may speculate as to how this might happen. In our model, the result of this settling is that the inner torus has a high density and thus controls the configuration of the local field lines, rather than the other way round. For example, the weight of the particles over the equator may cause the field lines to sag, as in some dense solar quiescent prominences, and particles could return directly to the surface. Turbulence may also help recirculate cloud particles to the star.

The phase-independent nature of the wind is more difficult to understand. GH97 pointed to the two types of wind, one originating from the surface (magnetic pole), the second, an exo-magnetospheric wind originating at the outer edge of the torus. If the absorption contributions of the two wind components were equal in the blue wings, one would expect the fluxes of their blue wings to vary sinusoidally twice per cycle - yet this is not observed. However, even if the wind mass fluxes were comparable, it is likely that their observable contributions in the blue wings of the line profiles could still be unequal for a few reasons. For example, the exo-magnetospheric wind will be more likely to remain visible over a wide range of phases, both as a consequence of its inverse-square density dependence with distance and because its streamlines will arc backwards to maintain angular momentum conservation. These curved trajectories will cross our lines of sight over a variety of rotational phases as they go off to infinity, diluting any modulation of wind absorption at any particular phase. The key requirement of all such arguments is that the wind remains visible over long path lengths.

As outlined in Sect. 3, a necessary requisite of our picture of rotating magnetic B stars is that closed field loops divert the wind flow from the magnetic poles to the torus at the magnetic equator. In this picture stalled wind particles return to the magnetic caps and establish a large composition gradient. This does not occur in nonmagnetic stars because envelope mixing destroys composition gradients before they can be established. Also, similar processes may take place in hot $\mathrm{Bp}$ analogs (e.g., $\theta^{1}$ Ori C), but its wind is so strong that chemical separation cannot be expected to take place. The results of this paper suggest indirectly that composition gradients are destroyed in magnetic, pulsating stars, such as in $\beta$ Cep and HR 6684. Yet, there are fundamental puzzles in even this simple picture. For example, what is to be concluded about HR 6684, which has low-metal but normal-He abundances? Perhaps the metals leave this star in the wind while both hydrogen and helium atoms are reaccreted. Such circumstances are envisioned by Hunger \& Groote (1999), but only in latetype B stars. A second puzzle is the Be-like episodes exhibited by $\beta$ Cep perhaps every 30 years (Kaper \& Mathias 1995; Panko \& Tarasov 1997). The tight scatter in the resonance line absorptions in Figs. 4a and in 5 of Henrichs et al. (2001) suggests that the presence or absence of a flattened Be disk does not affect torus-cloud signatures noticeably. Thus, if these ejections are related to the Bephenomenon, the UV phenomenology of the two types of circumstellar structures is vastly different (in addition to their physical morphologies).

In Sects. 1 and 5 we listed 13 probable members of the rotating magnetic B-star group (including $\theta^{1}$ Ori C). Rotating magnetic B stars appear also to include an indeterminate number of early-B main sequence stars with conditions that promote envelope mixing (pulsation, rapid rotation). One such example may be the unusual star 
HD 144941 (see also GH97). This star lies within the region of the $\mathrm{H}-\mathrm{R}$ Diagram populated by the He-strong stars and shows extreme He-richness and metal deficiencies. Its high galactic latitude, and high radial velocity $\left(-52.6 \mathrm{~km} \mathrm{~s}^{-1}\right)$ led Harrison \& Jeffery $(1997 \mathrm{a}, 1997 \mathrm{~b})$ and others to consider it as a member of the hydrogen-deficient B star class. Moreover, Harrison \& Jeffery (1997b) found that the Fe abundance is just as deficient as the CNO elements and suggested that these abundances are primordial. To test this result, we inspected two high-dispersion $I U E$ spectra obtained for this star reveal redshifted emissions in both the CIV and Si IV lines as well as a strong wind. These spectra show anomalously strong N V absorption so perhaps HD 144941 is a member too. We must also remark that the search for magnetic and rotational periods is very labor and telescope intensive, so new members of this class might be found in the long run by examining spectra of early-B stars for the presence of anomalous $\mathrm{N} \mathrm{V}$ absorptions and/or Si IV, C IV, N V emissions.

The physical attributes of the tori around the rotating magnetic stars can also be used to clarify our understanding of classical stars. Several studies (see Balona 2000) have suggested that co-rotating clouds are responsible for periodic spectroscopic and/or photometric varations in these stars. The difficulty in distinguishing between this explanation and nonradial pulsation is that optically thick clouds can mimic cool regions on the atmosphere. We are optimistic that some of the results in this paper that indicate the presence of redshifted components in resonance lines, the selective variation of low-excitation lines, and high turbulence together can help to discriminate between models responsible for the variability of these stars.

A general understanding of physical conditions leading to co-rotating magnetospheres will be aided by future studies of the magnetic rotating $B$ variables (with the probable extension to the sn He-weak stars (see Shore et al. 1987, 1990), hot He-strong analogues like $\theta^{1}$ Ori C, Ap stars like IQ Aur (Babel \& Montmerle 1997b), magnetic Herbig Ae-Be stars, and late-type active stars such as AB Dor. The results of this paper point to a number of desiderata which will clarify the nature of the class of rotating magnetic B stars in particular and also contribute to its global understanding. We close by listing a few of them:

- the IUE archive can be conveniently investigated to search for additional examples of early-B type stars with anomalous N V absorptions and redshifted emissions among its C IV and N V resonance lines;

- far-UV snapshot observations can be undertaken to determine whether the anomalous absorptions and redshifted emissions extend to the O VI doublet;

- the X-ray fluxes from these stars can be monitored around their rotational cycles to shed some light on the production mechanism of the CIV, N V absorptions;

- optical observations of the high Balmer lines can be carried out over rotational phase among all the candidate members of this class with a view of establishing whether cloud density varies with time and as a function of relevant stellar parameters;

- spectral imaging can profitably be carried out in the $\mathrm{H} \alpha$ line and ultimately in the strong ultraviolet resonance lines from space-borne interferometers.

Acknowledgements. We wish to thank the invisible author, Dr. Ivan Hubeny, for his making his suite of codes available for public access and for answering a number of questions we had about their efficient use. We also acknowledge an IDL wrapper for CIRCUS written by Dr. Richard Robinson for another study that permitted us to exploit the flexibility of the Hubeny codes. It is our pleasure to thank Dr. Huib Henrichs for providing us with a preprint of recent Zeeman analysis work on $\beta$ Cep. We are grateful to Dr. Kurt Hunger, Drs. Steve Cranmer and David Bohlender for important discussions. We also thank Drs. Luis Balona and Kurt Hunger for a critical reading of the manuscript. We appreciate a number of very helpful comments made by the referee, Dr. Steven Shore. This work was largely supported through NASA contracts NASA NAG 5-6733, 5-8793 and GO-6086.01-94A.

\section{References}

Babel, J. 1998, in Variable \& Non-spherical Stellar Winds, ed. B. Wolf, O. Stahl, \& A. Fullerton, IAU Colloq., No. 169 (Springer, Heidelberg), 187

Babel, J., \& Montmerle, T. 1996, in Hydrogen Deficient Stars, ed. C. Jeffery, \& U. Heber, ASP, San Francisco, 96, 189

Babel, J., \& Montmerle, T. 1997a, A\&A, 323, 121

Babel, J., \& Montmerle, T. 1997b, ApJ, 485, L29

Balona, L. A. 2000, in The Be Phenomenon in Early-Type Stars, ed. M. A. Smith, H. F. Henrichs, \& J. Fabregat, ASP Conf. Ser., 214, 1

Barker, P. K., Brown, D. G., Bolton, C. T., et al. 1982, in Advances in Ultraviolet Astronomy, ed. Y. Kondo, J. Mead, \& R. Chapman, NASA Conf. Pub., 2238, 442

Bohlender, D. A., Brown, D. N., Landstreet, J. D., et al. 1987, ApJ, 323, 325

Bohlender, D. A. 1994, in Pulsation, Rotation, \& Mass Loss in Early-Type Stars, ed. L. Balona, H. Henrichs, \& J. LeContel (Kluwer, Dordrecht), 185

Bolton, C. T., Fullerton, A. W., Bohlender, D. A., et al. 1986, in The Physics of Be Stars, ed. A. Slettebak, \& T. Snow (Cambridge Univ. Press, Cambridge), 82

Bolton, C. T. 1994, Ap. \& Sp. Sci., 221, 95

Brandt, J. C., Heap, S. R., Beaver, E. A., et al. 1998, AJ, 116, 941

Campos, A. J., \& Smith, M. A. 1980, ApJ, 238, 250

Cohen, D. H., MacFarlane, J. J., \& Wang, P. 1994, ApJ, 437, 351

Cohen, D. H., Finley, J. P., \& Cassinelli, J. P. 2000, ApJ, in press

Drake, S. A., Abbot, D. C., Bastian, T. S., et al. 1987, ApJ, 322,902

Drake, S. A., Linsky, J. L., Schmitt, J. H. M. M., et al. 1994, ApJ, 420, 387

Drake, S. A. 1998, Contr. Ast. Obsy. Pleso, 27, 382

Drew, J. 1989, ApJS, 71, 267

Fischel, D., \& Sparks, W. M. 1980, in The Universe at Ultraviolet Wavelengths, ed. R. D. Chapman, NASA Conf. Pub., 2171, 217 
Gagné, M., Caillault, J.-P., \& Stauffer, J. R. 1997, ApJ, 478, L87

Gies, D. R., \& Lambert, D. L. 1992, ApJ, 387, 673

Grigsby, J. A., \& Morrisson, N. D. 1995, ApJ, 442, 794

Groote, D., \& Hunger, K. 1976, A\&A, 52, 303

Groote, D., \& Hunger, K. 1982, A\&A, 116, 64

Groote, D., \& Hunger, K. 1997, A\&A, 319, 250 (GH97)

Havnes, O., \& Goertz, C. K. 1984, A\&A, 138, 421

Harrison, P. M., \& Jeffery, C. S. 1997a, A\&A, 323, 177

Harrison, P. M., \& Jeffery, C. S. 1997b, A\&A, 323, 393

Henrichs, H. F., de Jong, J. A., Nichols, J. S., et al. 1998, Ultraviolet Astrophysics Beyond the IUE Final Archive, ed. R. Harris (ESA SP-413) (Noordwijk: ESA), 157

Henrichs, H. F., de Jong, J. A., Donati, J.-F., et al. 2001, A\&A, submitted

Hesser, J. E., Moreno, H., \& Ugarte, P. P. 1977, ApJ, 216, L31

Hubeny, I. 1996, Complete Guide to Generate Stellar Spectra under the Influence of Absorbing and/or Emitting Bodies, priv. comm.

Hubeny, I., \& Heap, S. R. 1996, ApJ, 470, 1144

Hubeny, I., Lanz, T., \& Jeffery, S. 1994, Newslett. Anal. Astron. Spectra, 20, 30

Hunger, K., Heber, U., \& Groote, D. 1990, in Properties of Hot Luminous Stars, ed. C. D. Garmany, ASP. Conf. Ser., 7, 307 (HHG90)

Hunger, K., \& Groote, D. 1993, in Peculiar Verus Normal Phenomena in A-Type and Related Stars, ed. M. Dworetsky, F. Castelli, \& R. Faraggiana, ASP Conf. Ser., 44,394

Hunger, K., \& Groote, D. 1999, A\&A, 351, 554

Jerzykiewicz, M. 1972, PASP, 84, 718

Kaper, L., \& Mathias, P. 1995, in Astrophsyical Applications of Stellar Pulsation, ed. R. S. Stobie, \& P. A. Whitelock, ASP Conf. Ser., 83, 295

Kubiak, M., \& Seggewiss, W. 1984, Acta Astron., 34, 41

Kurucz, R. L. 1990, Trans. IAU, 20B, 169

Kurucz, R. L. 1993, ATLAS9 Stellar Atmospheres and 2 km s${ }^{-1}$ Grids, Kurucz CD-ROM \#13

Landstreet, J. D., \& Borra, E. F. 1978, ApJ, 224, L5
Linsky, J. L., Drake, S. A., \& Bastian, T. S. 1992, ApJ, 393, 341

Michaud, G., Dupuis, J., Fontaine, G., et al. 1987, ApJ, 322, 302

Millar, C. A., \& Marlborough, J. M. 1999, ApJ, 516, 276

Panko, E. A., \& Tarasov, A. E. 1997, Astron. Lett., 23, 545

Phillips, R. S., \& Lestrade, J.-F. 1988, Nature, 334, 329

Reiners, A., Stahl, O., Wolf, B., et al. 2000, A\&A, 363, 585

Shore, S. N. 1987, AJ, 94, 731

Shore, S. N. 1990, Evolution in Astrophysics, ed. J. Clavel, \& E. J. Rolfe, ESA SP-310, 501

Shore, S. N., \& Adelman, S. J. 1981, Chemically Peculiar Stars of the Upper Main Sequence, 23rd Liege Astrophysical Symposium (Univ. Liège, Liège), 429

Shore, S. N., \& Brown, D. N. 1990, ApJ, 365, 665 (SB90)

Shore, S. N., Brown, D. N., \& Sonneborn, G. 1987, AJ, 94, 737

Shore, S. N., Brown, D. N., Sonneborn, G., et al. 1990, ApJ, 348,242

Shore, S. N., \& Brown, D. N. 1998, in Variable \& Non-spherical Stellar Winds, ed. B. Wolf, O. Stahl, \& A. Fullerton, Op. Cit., 178

Short, I., \& Bolton, C. T. 1994, in Pulsation, Rotation \& Mass Loss in Early-Type Stars, ed. L. Balona, H. Henrichs, \& J. LeContel (Kluwer, Dordrecht), 171

Smith, M. A. 1999, PASP, 111, 722

Sparks, W. M., \& Fischel, D. 1971, Partition Functions and Equations of State in Plasmas, NASA SP-3066

Springmann, U. W., \& Pauldrach, A. W. 1992, A\&A, 262, 515

Vauclair, S., Dolez, N., \& Gough, D. O. 1991, A\&A, 252, 618

Wade, G. A., Bohlender, D. A., Brown, D. N., et al. 1997, A\&A, 320, 172

Walborn, N. R., Nichols-Bohlin, J., \& Panek, R. J. 1985, IUE Atlas of O-type Spectra from 1200 to $1900 \AA$, NASA RP-1155

Walborn, N. R., \& Nichols, J. S. 1994, ApJ, 425, L29

Walborn, N. R., Parker, J. M., \& Nichols, J. S. 1995, IUE Atlas of B-type Spectra from 1200 to $1900 \AA$, NASA Ref. Pub., No. 1363 